\title{
Connected chord diagrams and bridgeless maps
}

\author{
Julien Courtiel* \\ Université de Caen Normandie \\ ENSICAEN, CNRS, Laboratoire GREYC \\ Caen, France \\ julien.courtiel@unicaen.fr \\ Karen Yeats ${ }^{\dagger}$ \\ Department of Combinatorics and Optimization \\ University of Waterloo \\ Waterloo, ON, Canada \\ kayeats@uwaterloo.ca \\ Noam Zeilberger \\ School of Computer Science \\ University of Birmingham \\ Birmingham, United Kingdom \\ zeilbern@cs.bham.ac.uk
}

Submitted: Oct 17, 2017; Accepted: Sep 3, 2019; Published: Nov 22, 2019

(C) The authors. Released under the CC BY-ND license (International 4.0).

\begin{abstract}
We present a surprisingly new connection between two well-studied combinatorial classes: rooted connected chord diagrams on one hand, and rooted bridgeless combinatorial maps on the other hand. We describe a bijection between these two classes, which naturally extends to indecomposable diagrams and general rooted maps. As an application, this bijection provides a simplifying framework for some technical quantum field theory work realized by some of the authors. Most notably, an important but technical parameter naturally translates to vertices at the level of maps. We also give a combinatorial proof to a formula which previously resulted from a technical recurrence, and with similar ideas we prove a conjecture of Hihn. Independently, we revisit an equation due to Arquès and Béraud for the generating function counting rooted maps with respect to edges and vertices, giving a new bijective interpretation of this equation directly on indecomposable chord diagrams, which moreover can be specialized to connected diagrams and refined to incorporate the number of crossings. Finally, we explain how these results have a simple application to the combinatorics of lambda calculus, verifying the conjecture that a certain natural family of lambda terms is equinumerous with bridgeless maps.
\end{abstract}

Mathematics Subject Classifications: 05A19, 81T18

\footnotetext{
*Supported by the French "Agence Nationale de la Recherche" MetAConC, and the French INS2I JCJC Grant "ASTEC".

†Supported by an NSERC Discovery grant.
} 


\section{Contents}

1 Introduction $\quad 2$

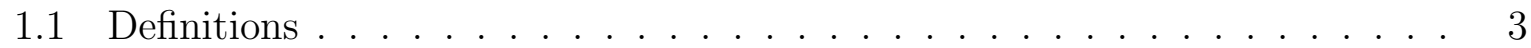

1.1.1 Chord diagrams . . . . . . . . . . . . . . . . . 3

1.1.2 Combinatorial maps ....................... 5

1.2 Enumerative and bijective links between maps and diagrams . . . . . . 7

1.3 Structure of the document . . . . . . . . . . . . . . . . . . 8

2 Equality of the cardinality sequences $\quad 8$

2.1 Between connected diagrams and bridgeless maps . . . . . . . . . . . . . . 9

2.2 Between indecomposable diagrams and maps . . . . . . . . . . . . . 10

3 Basic operations 11

3.1 Operations on chord diagrams . . . . . . . . . . . . . . . . 11

3.2 The Bridge First Labeling of a map . . . . . . . . . . . . . . . . . . . 13

3.3 Operations on maps . . . . . . . . . . . . . . . . . . . . . 14

4 Description of the main bijections $\quad 16$

4.1 Natural bijections . . . . . . . . . . . . . . . . . . . 16

4.2 Extension of $\theta$ and equality between bijections . . . . . . . . . . . . 17

4.3 Planar maps as diagrams with forbidden patterns . . . . . . . . . . 20

5 New perspectives on chord diagram expansions in QFT 22

5.1 Context: Feynman graphs and Dyson-Schwinger equations . . . . . . . . . 23

5.2 Context: chord diagram expansions of Dyson-Schwinger equations . . . . . 26

5.3 Diagram parameters and binary trees . . . . . . . . . . . . . 30

5.4 New interpretations on chord diagrams of the quantum field theoretic parameters . . . . . . . . . . . . . . . . . 33

5.5 Changing the ordering of the chords . . . . . . . . . . . 36

5.6 Restating the quantum field theory formulas in terms of maps . . . . . . . 40

5.7 A new combinatorial interpretation of a quantum field theoretic formula . . 44

6 New interpretation of the Arquès-Béraud functional equation $\quad 48$

6.1 Statement of the equation and implications . . . . . . . . . . . . 48

6.2 Combinatorial interpretation . . . . . . . . . . . . . . . 50

6.3 An application to lambda calculus . . . . . . . . . . . . . . . . 52

7 Conclusion $\quad 54$

\section{Introduction}

Connected chord diagrams are well-studied combinatorial objects that appear in numerous mathematical areas such as knot theory [27, 5, 33], graph sampling [1], analysis of data 
structures [12], and bioinformatics [16]. Their counting sequence (Sloane's A000699) has been known since Touchard's early work [29]. In this paper we present a bijection with another fundamental class of objects: bridgeless combinatorial maps. Despite the ubiquity of both families of objects in the literature, this bijection is, to our knowledge, new. Furthermore, it is fruitful in the sense that it generalizes and restricts well, and useful parameters carry through it.

\subsection{Definitions}

Before outlining the contributions of the paper more precisely, we begin by recalling here the formal definitions of (rooted) chord diagrams and (rooted) combinatorial maps, together with some auxiliary notions and notation.

The reader already familiar with these notions may jump straight to Sections 1.2 and 1.3 to find a detailed presentation of our results.

\subsubsection{Chord diagrams}

Definition 1 (Matchings on linear orders). Let $P$ be a linearly ordered finite set. An $n$-matching in $P$ is a mutually disjoint collection $C$ of ordered pairs $\left(a_{1}, b_{1}\right), \ldots,\left(a_{n}, b_{n}\right)$ of elements of $P$, where $a_{i}<b_{i}$ for each $1 \leqslant i \leqslant n$. A perfect matching in $P$ is a matching which includes every element of $P$.

Definition 2 (Chord diagrams). A rooted chord diagram is a linearly ordered, non-empty finite set $P$ equipped with a perfect matching $C$. The pairs in $C$ are called chords, while the root chord is the unique pair whose first component is the least element of $P$.

Two $n$-matchings $(P, C)$ and $\left(P^{\prime}, C^{\prime}\right)$ are considered isomorphic if they are equivalent up to relabeling of the elements and reordering of the pairs, or in other words, if there is an order isomorphism $\phi: P \cong P^{\prime}$ and a permutation $\pi \in S_{n}$ such that $\phi C=C^{\prime} \pi$, where $\phi C=\left(\phi\left(a_{1}\right), \phi\left(b_{1}\right)\right), \ldots,\left(\phi\left(a_{n}\right), \phi\left(b_{n}\right)\right)$ denotes the image of $C$ under $\phi$, and $C^{\prime} \pi=$ $\left(a_{\pi(1)}^{\prime}, b_{\pi(1)}^{\prime}\right), \ldots,\left(a_{\pi(n)}^{\prime}, b_{\pi(n)}^{\prime}\right)$ denotes the reindexing of $C^{\prime}$ by $\pi$. Up to isomorphism, a chord diagram with $n \geqslant 1$ chords may therefore be identified with a perfect matching on the ordinal $2 n=\{0<\cdots<2 n-1\}$, and so we will usually omit reference to the underlying set of a chord diagram, simply keeping track of the number of chords $n$ (we refer to the latter as the size of the diagram). Isomorphism classes of chord diagrams of size $n$ can also be presented as fixed point-free involutions on the set $2 n$, although we find the definition as a perfect matching more convenient to work with.

To visualize a chord diagram, we represent the elements of its underlying linear order by a series of collinear dots, and the matching by a collection of arches joining the dots together in pairs: see Figure 1(a) for an example. In the literature, rooted chord diagrams are also drawn according to a circular convention: instead of being arranged on a line, the $2 n$ points are drawn on an oriented circle and joined together by chords, and then one point is marked as the root. This convention has been notably used in $[22,15]$, but the linear convention is the one we adopt for the rest of the document ${ }^{1}$.

\footnotetext{
${ }^{1}$ People also consider unrooted chord diagrams with no marked point, see for example [20, §6.1]. Since
} 
(a)

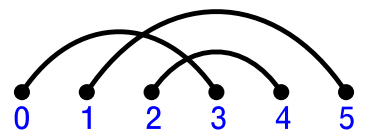

(b)

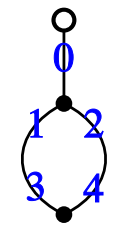

$$
\begin{aligned}
H & =\{0,1,2,3,4\} \\
\sigma & =(012)(34) \\
\alpha & =(0)(13)(24)
\end{aligned}
$$

\begin{tabular}{|c|c|c|c|}
\hline Objects & Size 1 & Size 2 & Size 3 \\
\hline $\begin{array}{l}\text { Connected } \\
\text { diagrams }\end{array}$ & $\curvearrowright$ & $m$ & $m \times A M A M$ \\
\hline $\begin{array}{c}\text { Bridgeless } \\
\text { maps }\end{array}$ & & & \\
\hline
\end{tabular}

Figure 1: (a) Rooted chord diagram associated to the perfect matching $(0,3),(1,5),(2,4)$. (b) A rooted map and its permutation representation.

Table 1: Small connected diagrams and bridgeless maps

Definition 3 (Intersection graph, connected diagrams). The intersection graph of a chord diagram $C$ is defined as the digraph with a vertex for every chord, and an oriented edge from chord $(a, b)$ to chord $(c, d)$ whenever $a<c<b<d$. A chord diagram is said to be connected (or irreducible) if its intersection graph is (weakly) connected.

Equivalently, a diagram of size $n$ is connected if for every proper non-empty subsegment $[i, j] \subset[0,2 n-1]$, there exists a chord with one endpoint in $[i, j]$ and the other endpoint outside $[i, j]$. All connected diagrams of size $\leqslant 3$ are depicted in the first row of Table 1 . Besides connectedness, we also consider the weaker notion of "indecomposability" of a diagram, defined in terms of diagram concatenation.

Definition 4 (Diagram concatenation). Let $C_{1}$ and $C_{2}$ be chord diagrams of sizes $n_{1}$ and $n_{2}$, respectively. The concatenation of $C_{1}$ and $C_{2}$ is the chord diagram $C_{1} C_{2}$ of size $n_{1}+n_{2}$ whose underlying linear order is given by the ordinal sum of the underlying linear orders of $C_{1}$ and $C_{2}$, and whose matching is determined by $C_{1}$ on the first $2 n_{1}$ elements and by $C_{2}$ on the next $2 n_{2}$ elements.

As the name suggests, diagram concatenation has a simple visual interpretation as laying two chord diagrams side by side.

Definition 5 (Indecomposable diagrams). A rooted chord diagram is said to be indecomposable if it cannot be expressed as the concatenation of two smaller diagrams.

Every connected diagram is indecomposable, but the converse is not true: see Table 2.

we work only with rooted chord diagrams in this paper, we refer to them simply as chord diagrams, or even as "diagrams" when there is no confusion. 


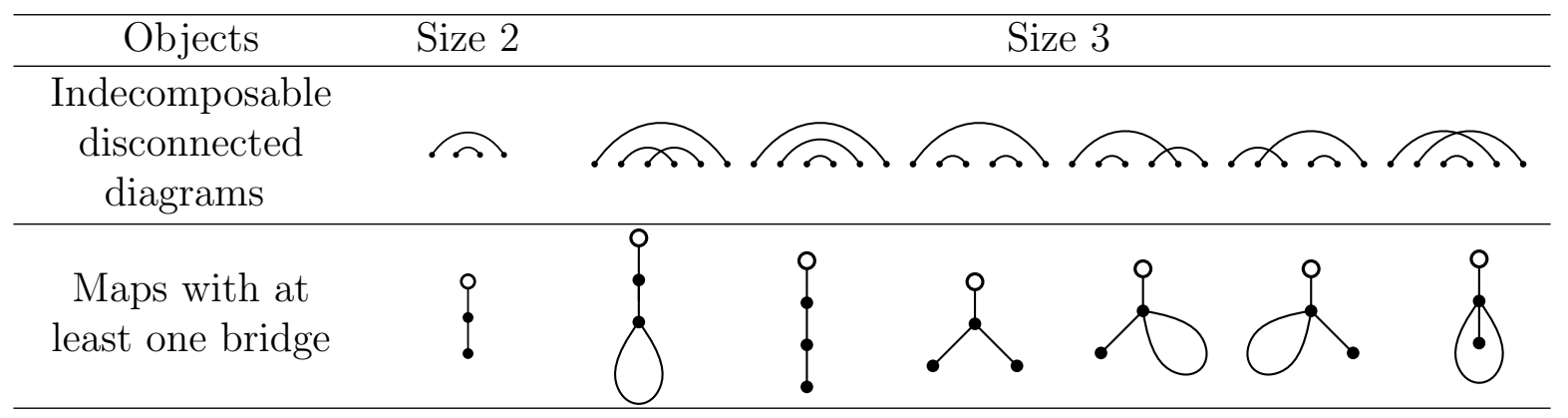

Table 2: Small indecomposable diagrams and maps not displayed in Table 1.

Finally, it will often be convenient for us to speak about intervals in a chord diagram. By an interval, we simply mean a pair of successive points: thus a diagram with $n$ chords (joining $2 n$ points) has $2 n-1$ intervals.

\subsubsection{Combinatorial maps}

Combinatorial maps are representations of embeddings of graphs into oriented surfaces $[19,20,11]$. Like chord diagrams, they come in both rooted and unrooted versions, but we will be dealing only with rooted maps in this paper.

Definition 6 (Combinatorial maps). A rooted combinatorial map is a set equipped with a transitive action of the group $\Gamma=\left\langle\sigma, \alpha \mid \alpha^{2}=1\right\rangle$ and a distinguished fixed point for the action of $\alpha$. Explicitly, this consists of the following data:

- a set $H$ (whose elements are called half-edges);

- a permutation $\sigma$ and an involution $\alpha$ on $H$;

- a half-edge $r \in H$ (called the root) for which $\alpha(r)=r$;

- such that between any pair of half-edges $x, y \in H$, there is a permutation $f$ defined using only compositions of $\sigma$ and $\alpha$ (and/or their inverses) for which $f(x)=y$.

Two rooted combinatorial maps are considered isomorphic just when there is a bijection between their underlying sets of half-edges which commutes with the action of $\Gamma$ and preserves the root. Note that our definition of combinatorial maps is a bit non-standard in allowing the involution $\alpha$ to contain fixed points and taking the root as a distinguished fixed point of $\alpha$. Defining the root as a fixed point is convenient for dealing with the trivial map (pictured at the left end of the second row of Table 1), while the presence of additional fixed points means that in general our maps can have "dangling edges" in addition to the root. Formally, the underlying graph of a combinatorial map is defined as follows.

Definition 7 (Underlying graph). Let $M=(H, \sigma, \alpha, r)$ be a rooted combinatorial map. The underlying graph of $M$ has vertices given by the orbits of $\sigma$, edges given by the orbits of $\alpha$, and the incidence relation between vertices and edges defined by their intersection. 
For any $v \in \operatorname{orbit}(\sigma)$ and $e \in \operatorname{orbit}(\alpha)$ we have $|v \cap e| \in\{0,1,2\}$, that is, a vertex and an edge can be incident either zero, once, or twice in the underlying graph. An edge which is incident to the same vertex twice is called a loop, while an edge which is incident to only one vertex exactly once is called a dangling edge. The size of a map is defined here as the number of edges in its underlying graph (giving full value to dangling edges). We call a combinatorial map closed if its underlying graph contains no dangling edges other than the root, and otherwise we call it open. For the most part, we will be dealing with closed maps, so we usually omit the qualifier unless it is important to remind the reader when we are dealing with open maps (as will at times be convenient). We also usually omit the prefix "rooted", again because we only ever consider rooted combinatorial maps.

Figure 1(b) shows an example of a (closed rooted) combinatorial map and its graphical realization, where we have indicated the unattached end of the root by a white vertex. This is also an example of a bridgeless map in the sense of Definition 9 below.

Proposition 8. The underlying graph of any combinatorial map is connected.

Proof. By transitivity of the action of $\Gamma$.

Definition 9 (Bridgeless maps). A combinatorial map is said to be bridgeless if its underlying graph is 2-edge-connected, that is, if there does not exist an edge whose deletion separates the graph into two connected components (such an edge is called a bridge).

The second row of Table 1 lists all (closed) bridgeless maps with at most three edges, while the second row of Table 2 lists all the remaining maps of size $\leqslant 3$. Observe that although the half-edges are unlabeled (again, since we are interested in isomorphism classes of labelled structures), the specification of the permutation $\sigma$ is contained implicitly in the cyclic ordering of the half-edges around each vertex, and the specification of the involution $\alpha$ in the gluing together of half-edges to form edges. Observe also that one of the maps in Table 1 contains a pair of crossing edges: such crossings should be thought of as "virtual", arising from the projection of a graph embedded in a surface of higher genus down to the plane. For a more detailed discussion of the precise correspondence between combinatorial maps and embeddings of graphs into oriented surfaces, see [19, 20, 11].

Finally, we introduce a few additional technical notions. In a rooted map, we distinguish the root from the root edge and the root vertex: the root vertex is the unique vertex which is incident to the root, while the root edge (in a map of size $>1$ ) is the unique edge following the root in the positive direction (i.e., according to the permutation $\sigma$ ) around the root vertex. A corner is the angular section between two distinct adjacent half-edges. The root corner is the corner between the root and the root edge. Half-edges are in obvious bijection with corners (for maps of size $>1$ ), but it is often more convenient to work with the corners: for example, pointing out two corners is a clear way to show how to insert an edge in a map. 


\subsection{Enumerative and bijective links between maps and diagrams}

Expanding definitions, it is not hard to see that one-vertex combinatorial maps are in direct correspondence with chord diagrams:

$$
\text { [one-vertex combinatorial maps] } \longleftrightarrow \text { [chord diagrams]. }
$$

Indeed, any (closed) one-vertex map determines a fixed point-free involution $\alpha$ on its non-root half-edges, together with a linear order induced by the cycle $\sigma$.

From a completely different direction, we demonstrate in this paper the existence of a size-preserving bijection between bridgeless maps (with arbitrarily many vertices) and connected diagrams:

$$
\text { [bridgeless combinatorial maps] } \stackrel{\theta}{\longleftrightarrow} \text { [connected chord diagrams]. }
$$

Indeed, we prove that $\theta$ is the restriction of a bijection between general combinatorial maps and indecomposable diagrams:

$$
\text { [combinatorial maps] } \stackrel{\phi}{\longleftrightarrow} \text { [indecomposable chord diagrams]. }
$$

Conversely, we also prove that $\phi$ is the extension of $\theta$ obtained by composing with a canonical decomposition of rooted maps (respectively, indecomposable diagrams) in terms of the bridgeless (respectively, connected) component of the root.

The existence of $\theta$ implies the following enumerative statement.

Theorem 10. The number of rooted bridgeless combinatorial maps of size $n$ is equal to the number of rooted connected chord diagrams of size $n$.

The fact that bridgeless maps and connected diagrams define equivalent combinatorial classes has apparently not been previously observed in the literature, let alone with a bijective proof. On the other hand, an explicit bijection between combinatorial maps and indecomposable diagrams was already given by Ossona de Mendez and Rosenstiehl $[24,25]$, who moreover wrote (in the early 2000s) that the corresponding enumerative statement "was known for years, in particular in quantum physics", although "no bijective proof of this numerical equivalence was known".

Theorem 11 (Ossona de Mendez and Rosenstiehl [24, 25]). The number of rooted combinatorial maps of size $n$ is equal to the number of rooted indecomposable chord diagrams of size $n$.

It may appear surprising that Theorem 10 has been seemingly overlooked despite Theorem 11 having been "known for years", and with the latter even being given a nice bijective proof over a decade ago (that was further analyzed and simplified by Cori [8]). Yet, there is a partial explanation: it turns out that Ossona de Mendez and Rosenstiehl's bijection does not restrict to a bijection between bridgeless maps and connected maps - and moreover cannot for intrinsic reasons that we will discuss in Section 6.1. In other words, both of the bijections $\theta$ and $\phi$ we describe in this paper are apparently fundamentally new, and we will see that they have interesting applications. 


\subsection{Structure of the document}

We will begin in Section 2 by showing that connected diagrams and bridgeless maps are equinumerous due to them satisfying the same recurrences, and similarly for indecomposable diagrams and general maps. Implicitly this already induces bijections, but there are choices to be made, and good choices will give bijections preserving interesting and important statistics. Thus we will proceed in Section 3 to define operations on diagrams and maps which will be the building blocks of the bijections. The bijections themselves are presented in Section 4. Our bijection from connected diagrams to bridgeless maps has two descriptions, one of which makes clear that it extends to a bijection between indecomposable diagrams and general maps that we also give. Furthermore, we characterize those diagrams which are taken to planar maps under our bijection.

The remainder of the paper looks at applications resulting from our bijections. Section 5 applies our bijection from connected diagrams to some chord diagram expansions in quantum field theory which some of us, with other collaborators, have discovered as series solutions to a class of functional equations in quantum field theory. Some interesting results have been proved thanks to the diagram expansions, but some of the diagram parameters were obscure. We will use our bijections to maps to simplify and make more natural these parameters and the resulting expansion. Most notably, a special class of chords, known as terminal chords, corresponds to vertices in the maps. Moreover, we use this new interpretation in terms of maps to give a combinatorial proof to a quite involved formula appearing in [15], which was a key point of that article but did not have a clear explanation aside a technical recurrence, and with similar ideas we prove a conjecture of Hihn.

Section 6 revisits a functional equation of Arquès and Béraud for the generating function counting rooted maps with respect to edges and vertices. We give a new bijective interpretation of this functional equation directly on indecomposable chord diagrams, with the important property that it restricts to connected diagrams to verify a modified functional equation. These equations have also appeared recently in studies of the combinatorics of lambda calculus, and we explain how to use our results to verify a conjecture that a certain family of lambda terms is equinumerous with bridgeless maps.

\section{Equality of the cardinality sequences}

Once the observation has been made, it is quite elementary to show that the cardinalities of the above-mentioned classes are the same by proving that they satisfy the same recurrences, as we will do in this section. First, we establish the recurrence for connected diagrams and bridgeless maps, which implies Theorem 10. Then, we establish a recurrence for indecomposable diagrams and unrestrained maps, which yields a new proof of Theorem 11. Note that the propositions we prove in this section also yield implicit correspondences between the combinatorial classes, but they do not determine which map a given diagram must be sent to. Although it is easy to settle that in an arbitrary way, the more careful analysis of Section 3 and 4 will yield bijections preserving various important 
statistics.

\subsection{Between connected diagrams and bridgeless maps}

We combinatorially show the following recurrence - which characterizes the sequence A000699 in the OEIS - for connected diagrams and bridgeless maps. The formula was given by Stein [26] for connected diagrams, but it has never been stated for bridgeless maps.

Proposition 12. The number $c_{n}$ of rooted connected diagrams of size $n$ and the number of rooted bridgeless maps of size $n$ both satisfy $c_{1}=1$ and

$$
c_{n}=\sum_{k=1}^{n-1}(2 k-1) c_{k} c_{n-k} .
$$

Proof. The recurrence relation translates the fact that it is possible to combine two objects, one of which is weighted by twice its size (minus 1), to bijectively give a bigger object of cumulated size. We describe how to do so for our two classes.
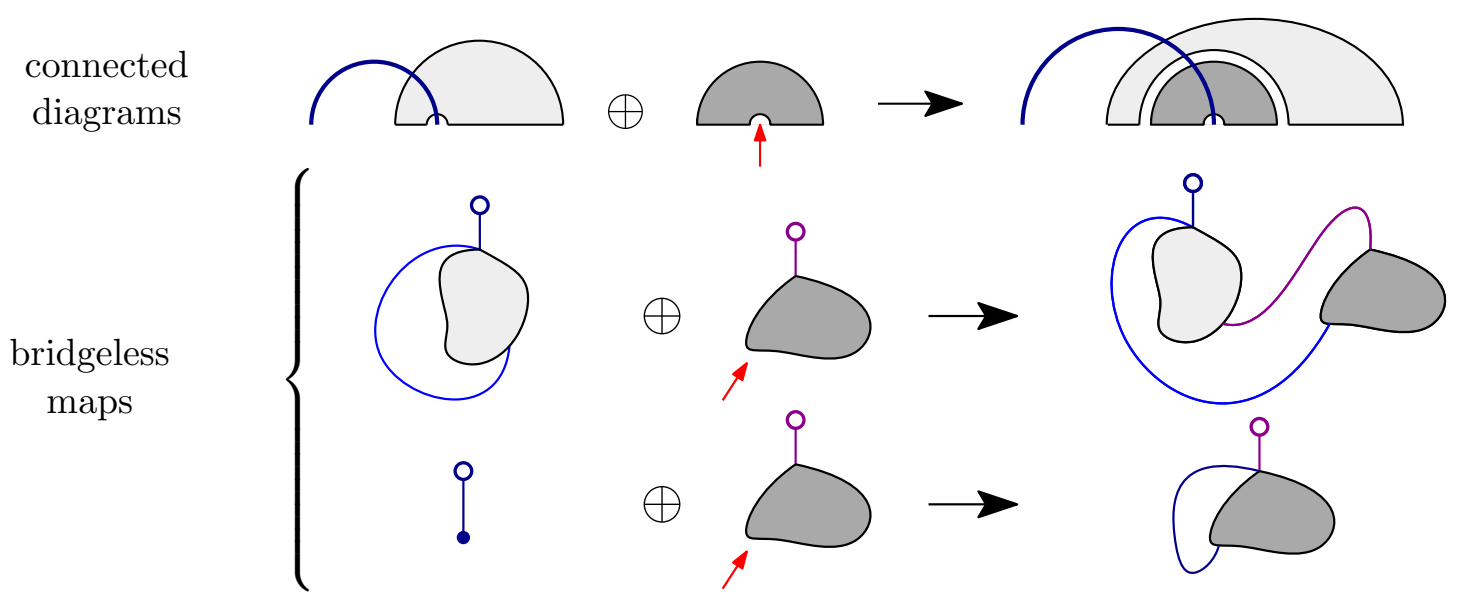

Figure 2: Schematic decomposition of connected diagrams and bridgeless maps.

Connected diagrams. For connected diagrams, $2 k-1$ counts the number of intervals delimited by $k$ chords. In other words, it means there are $2 k-1$ ways to insert a new root chord in a diagram of size $k$. We can find in the literature numerous ways to combine a diagram $C_{1}$ with another diagram $C_{2}$ with a marked interval [23]. The one we choose comes from [10] and is illustrated in Figure 2. The idea is to insert $C_{2}$ into $C_{1}$, just after the root chord of $C_{1}$. Then, we move the right endpoint of the root chord of $C_{1}$ to the marked interval of $C_{2}$. We thus obtain our final combined diagram.

To recover $C_{1}$ and $C_{2}$, we mark the interval just after the root chord. Then, we pull the right endpoint of the diagram to the left until the diagram disconnects into two connected components. The first component is $C_{1}$, the second one $C_{2}$. 
Bridgeless maps. In maps of size $k$, the number $2 k-1$ refers to the number of corners. Given two maps $M_{1}$ and $M_{2}$ where $M_{2}$ has a marked corner, we construct a larger map as follows (this is also illustrated in Figure 2).

If $M_{1}$ has size 1 , we insert a new edge in $M_{2}$ which links the root corner of $M_{2}$ to its marked corner. If $M_{1}$ has size greater than 1 then it has a root edge. Let us unstick the second endpoint of the root edge and insert it in the marked corner of $M_{2}$. Then, we take the root of $M_{2}$ and insert it where the second endpoint of the root edge of $M_{1}$ was. We thus obtain our final map. Note that no bridge has been created in the process.

To recover $M_{1}$ and $M_{2}$, we start by marking the corner after the second endpoint of the root edge of the new map. Then, grab this endpoint and slide it up, towards the root. When a bridge appears, we stop the process and cut the bridge, marking it as a root. The two resulting diagrams are $M_{1}$ are $M_{2}$. If we reach the root vertex with this process without creating any bridge, then it means that $M_{1}$ was the trivial map with one half-edge. In that case, we obtain $M_{2}$ by just removing the root edge.

\subsection{Between indecomposable diagrams and maps}

We now prove a similar proposition for indecomposable diagrams and unconstrained maps. The recurrence formula, which we can consider to be part of folklore, is essentially the one described by Arquès and Béraud for maps [2]. To our knowledge, this has never been combinatorially interpreted for indecomposable diagrams.

Proposition 13. The number $b_{n}$ of indecomposable diagrams of size $n$ and the number of rooted maps of size $n$ both satisfy $b_{1}=1$ and

$$
b_{n}=\sum_{k=1}^{n-1} b_{k} b_{n-k}+(2 n-3) b_{n-1} .
$$

Proof. The decompositions for both classes, which we describe in this proof, are illustrated by Figure 3.

Indecomposable diagrams. For an indecomposable diagram $D$ of size $n>1$, there are two exclusive possibilities.

- The deletion of the root chord makes the diagram decomposable, i.e. the resulting diagram is the concatenation of several indecomposable diagrams. Let $D_{1}$ be the first one of them, and $D_{2}$ the diagram $D$ where we have removed $D_{1}$ while leaving the root chord in place. The transformation is reversible; we can recover $D$ from $D_{1}$ and $D_{2}$ by putting $D_{1}$ in the leftmost interval (after the left endpoint of the root chord) of $D_{2}$. Thus, if $D_{1}$ has size $k$, the number of such diagrams $D$ is $b_{k} b_{n-k}$.

- The deletion of the root chord induces another indecomposable diagram $\boldsymbol{D}^{\prime}$. Then $D^{\prime}$ has size $n-1$ and we can recover $D$ via a root chord insertion. As mentioned in the proof of Proposition 12, a chord diagram with $k$ chords has $2 k-1$ 


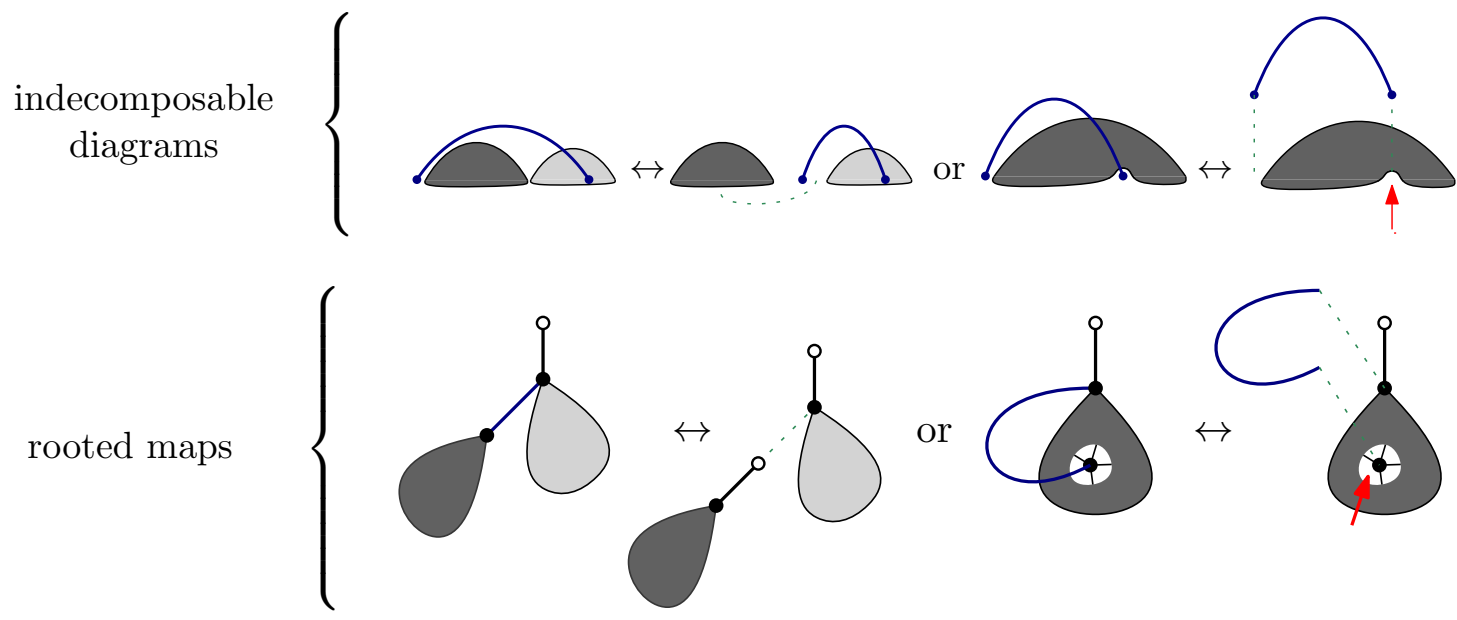

Figure 3: Schematic decomposition of indecomposable diagrams and maps.

intervals, so there are $2 n-3$ different ways to insert a root chord in $D^{\prime}$. Thus, the number of such diagrams is $(2 n-3) b_{n-1}$.

The conjunction of both cases gives Equation 2.

Maps. The decomposition we give is based on Tutte's classic root edge removal procedure, extended to the arbitrary genus case $[2,11]$. We distinguish again two exclusive possibilities for a rooted map of size $n>1$.

- The root edge is a bridge. In other words, $M$ joins two different maps $M_{1}$ and $M_{2}$ via a bridge. If $M_{1}$ has size $k$, there are then $b_{k} b_{n-k}$ such maps.

- The root edge is not a bridge. Then $M$ is obtained from a map of size $n-1$ by a root edge insertion. There are $2 n-3$ ways to insert a root edge in a map of size $n-1$ (this corresponds to the number of corners). Thus, the number of such maps is $(2 n-3) b_{n-1}$.

Again, Equation 2 results from the consideration of these two cases.

\section{Basic operations}

We define in this section several basic operations on chord diagrams and combinatorial maps, which will be used in Section 4 to formally construct bijections between connected diagrams and bridgeless maps, and between indecomposable diagrams and general maps.

\subsection{Operations on chord diagrams}

Definition 14 (Operations RootIns and DiagIns). Let $D$ be a diagram of size $n, k$ an integer $1 \leqslant k \leqslant 2 n-1$, and $D^{\prime}$ an arbitrary diagram. We write $\operatorname{RootIns}_{k}(D)$ to denote the diagram obtained from $D$ by inserting a new root chord whose right endpoint ends 
in the $k$ th interval of $D$ (from left to right), and $\operatorname{DiagIns}_{D^{\prime}, k}(D)$ to denote the diagram obtained from $D$ by inserting the diagram $D^{\prime}$ into the $k$ th interval of $D$. (Figure 4 shows examples of both operations.)

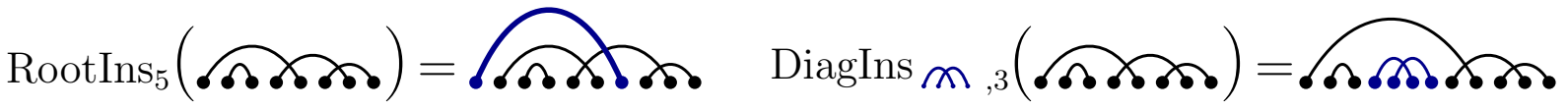

Figure 4: Illustration of operations RootIns and DiagIns.

The following technical lemma describes an important commutation relation between RootIns and DiagIns.

Lemma 15. Let $k$ and $\ell$ be two integers and $D$ an indecomposable chord diagram. We have the commutation rules

$$
\begin{array}{rr}
\operatorname{DiagIns}_{D, \ell} \circ \operatorname{RootIns}_{k}=\text { RootIns }_{k} \circ \operatorname{DiagIns}_{D, \ell-2}, & \text { if } k \leqslant \ell-2, \\
\operatorname{DiagIns}_{D, \ell} \circ \operatorname{RootIns}_{k}=\text { RootIns }_{k+2|D|} \circ \operatorname{DiagIns}_{D, \ell-1}, & \text { if } 1 \leqslant \ell-1 \leqslant k,
\end{array}
$$

where $|D|$ is the number of chords in $D$.

Proof. Each time we $(i)$ insert a new root chord into a diagram $C$ and then $(i i)$ insert a diagram into $C$, we can choose to do it in the opposite order - $(i i)$ then $(i)$ - as long as the diagram is not inserted into the first interval. The only things we have to take care of are the positions where the insertions occur, which can change after a root chord insertion or a diagram insertion. Thus, the $i$ th leftmost interval becomes, after an operation RootIns ${ }_{k}$, the $(i+1)$ th leftmost interval if $i<k$, and the $(i+2)$ th one if $i>k$. Similarly, after an operation DiagIns $_{D, \ell}$, the $i$ th leftmost interval remains the $i$ th leftmost interval if $i<\ell$, and will become the $(i+2|D|)$ th leftmost interval if $i>\ell$. Equations (3) and (4) follow from this analysis.

Finally, we define an operation on combinatorial objects, that we name indexed product. For connected diagrams, it corresponds to the combination of two connected diagrams described in the proof of Proposition 12.

Definition 16 (Indexed product for connected diagrams). Let $C_{1}$ and $C_{2}$ be two connected diagrams, and $i$ be an integer between 1 and $2\left|C_{2}\right|-1$, where $\left|C_{2}\right|$ is the size of $C_{2}$. The connected diagram $C_{1} \star_{i} C_{2}$ is defined as

$$
\begin{array}{r}
\operatorname{RootIns}_{i}\left(C_{2}\right) \\
\operatorname{RootIns}_{i+\ell}\left(\operatorname{DiagIns}_{C_{2}, \ell}\left(\widehat{C_{1}}\right)\right) \quad \text { if } C_{1} \text { is the one-chord diagram, }
\end{array}
$$

Examples of this operation are shown in Figure 5. Let us recall, as used in the proof of Proposition 12, that the star product induces a bijection between connected diagrams $C$, and triples $\left(C_{1}, C_{2}, i\right)$ where $C_{1}$ and $C_{2}$ are two connected diagrams, and $i \in\left\{1, \ldots, 2\left|C_{2}\right|-1\right\}$.

Other similar definitions are both possible and useful. We will define a variant of the indexed product for some technical work in Subsection 5.3 (see Definition 34). 


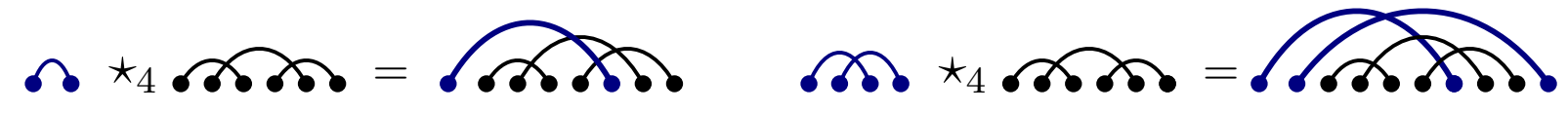

Figure 5: Illustration of the indexed product for connected diagrams.

\subsection{The Bridge First Labeling of a map}

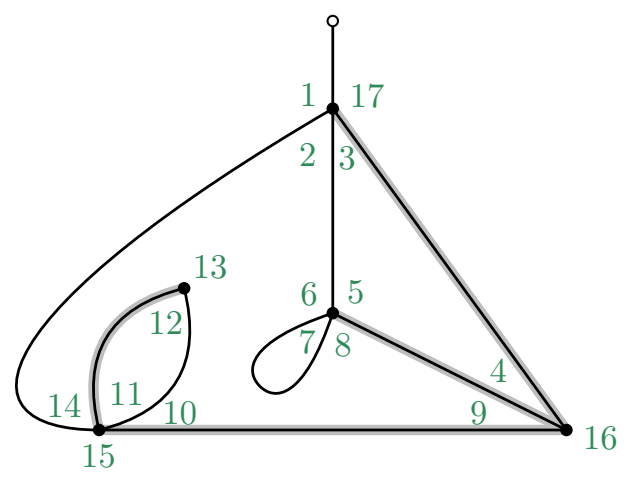

Figure 6: The Bridge First Labeling of a map. The overlined edges correspond to the edges which become bridges during the algorithm described in Subsection 3.2. Alternatively, they form the spanning tree associated to the rightmost DFS.

Given a rooted map $M$ (potentially with dangling edges), we describe in this subsection a way to label the corners of $M$, which we call the Bridge First Labeling of $M$. We choose this labeling because we want the operations of insertions in maps to satisfy an analogue of Lemma 15.

The Bridge First Labeling is given by the following algorithm.

- The first corner we consider is the root corner. We label it by 1 .

- Assume the current corner is labeled by $k$, and consider the (potentially dangling) edge $e$ adjacent to this corner in the counterclockwise order. There are three possibilities:

- The edge $e$ is a bridge. Go along this edge to the next corner. Label this corner by $k+1$.

- The edge $e$ is a dangling edge. Go to the following corner in the counterclockwise order, and label it by $k+1$.

- The edge $e$ is neither dangling nor a bridge. Cut $e$ into two dangling edges. Go to the following corner in the counterclockwise order, and label it by $k+1$.

- The algorithm stops when we reach the root.

An example of a run of this algorithm has been started in Figure 7. 

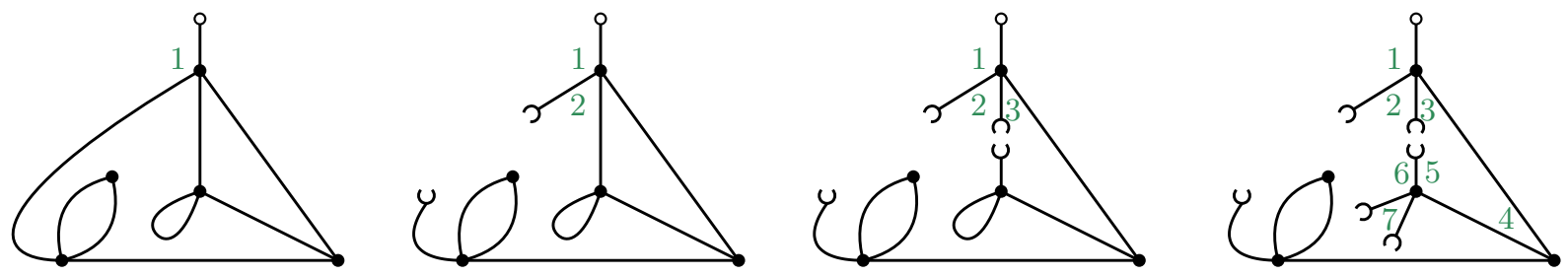

Figure 7: The first steps of the Bridge First Labeling of the map of Figure 6.

Alternatively, the Bridge First Labeling can be deduced from the tour ${ }^{2}$ of the spanning tree induced by the Depth First Search (DFS) of the map where we favor the rightmost edges (call this a rightmost DFS). The notion of rightmost DFS will return in Subsection 5.6.

\subsection{Operations on maps}

Now that we have set a suitable way to label the corners of a map, we define two analogues of RootIns and DiagIns for maps:

Definition 17 (Operations RootIns and MapIns). Let $M$ be a map of size $n, k$ an integer $1 \leqslant k \leqslant 2 n-1$, and $M^{\prime}$ an arbitrary map. We write $\operatorname{RootIns}_{k}(M)$ to denote the map obtained from $M$ by adding an edge linking the root corner and the $k$ th corner of the Bridge First Labeling of $M$. We write $\operatorname{MapIns}_{M^{\prime}, k}(M)$ to denote the insertion of $M^{\prime}$ in $M$ via a bridge at the $k$ th corner of the Bridge First Labeling of $M$.

Examples are given by Figure 8.
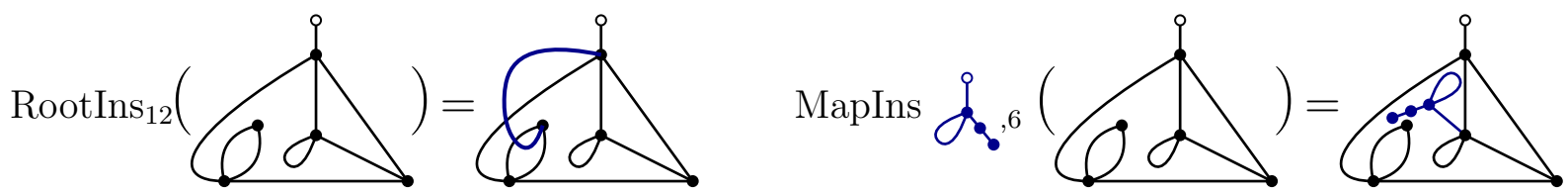

Figure 8: Illustration of operations RootIns and MapIns.

The next lemma explains why we have chosen the Bridge First Labeling as a canonical way to number the corners of a map: the operations RootIns and MapIns satisfy an analogous commutation relation as the corresponding operations RootIns and DiagIns on diagrams (Lemma 15). Numerous statistics will be thus preserved when we transform a map into a diagram.

Lemma 18. Let $k$ and $\ell$ be two integers, and $M$ be a combinatorial map (with only one

\footnotetext{
${ }^{2}$ in the sense of [3]: we visit every half-edge, starting by the root. If a half-edge does not belong to the spanning tree, we go to the next half-edge in counterclockwise order; it a half-edge does belong to it, we follow the associated edge.
} 
dangling edge, marking the root). We have the commutation rules

$$
\begin{aligned}
\text { MapIns }_{M, \ell} \circ \operatorname{RootIns}_{k}=\text { RootIns }_{k} \circ \operatorname{MapIns}_{M, \ell-2}, & \text { if } k \leqslant \ell-2, \\
\text { MapIns }_{M, \ell} \circ \operatorname{RootIns}_{k}=\operatorname{RootIns}_{k+2|M|} \circ \operatorname{MapIns}_{M, \ell-1}, & \text { if } 1 \leqslant \ell-1 \leqslant k,
\end{aligned}
$$

where $|M|$ is the number of edges in $M$.

Proof. Similarly as in Lemma 15, we have to understand how a root edge insertion or a diagram insertion affects the labels of a map.

The edge added by the operation RootIns $k$ will be necessarily cut in half at the start of the Bridge First Labeling algorithm. The rest of the tour will be unchanged, except for an extra step at the $k$ th position, which is the visit of the second dangling edge resulting from the root edge. Therefore, a corner labeled by $i$ with $i<k$ will carry the label $i+1$ (the first dangling edge has been visited but not the second one), while a corner labeled by $i$ with $i<k$ will carry the label $i+2$.

Concerning the operation MapIns $_{M, \ell}$, it will only affect the labels of the corners which are after $\ell$. Indeed, after the $\ell$ th step, we have to visit the entire map $M$, which counts $2|M|$ corners. Thus, a corner with label $i>\ell$ will carry the label $i+2|M|$ after the operation MapIns M, $\ell$

Finally, we define an indexed product for bridgeless maps. As for connected diagrams, this product describes the combination between two bridgeless maps which is stated in the proof of Proposition 12. It is the formal analog of Definition 16.

Definition 19 (Indexed product for bridgeless maps). Let $M_{1}$ and $M_{2}$ be two bridgeless maps, and $i$ an integer between 1 and $2\left|M_{2}\right|-1$, where $\left|M_{2}\right|$ is the size of $M_{2}$. The bridgeless map $M_{1} \star_{i} M_{2}$ is defined as

$$
\operatorname{RootIns}_{i}\left(M_{2}\right) \quad \text { if } M_{1} \text { is reduced to a root, }
$$

$\operatorname{RootIns}_{i+\ell}\left(\operatorname{MapIns}_{M_{2}, \ell}\left(\widehat{M}_{1}\right)\right) \quad$ if $M_{1}$ is of the form $\operatorname{RootIns}\left(\widehat{M}_{1}\right)$ for some $\widehat{M}_{1}$.

Once again, following the proof of Proposition 12, for each bridgeless map $M$ of size $>1$, there exists a unique triple $\left(M_{1}, M_{2}, i\right)$ where $M_{1}$ and $M_{2}$ are two bridgeless maps such that $M=M_{1} \star_{i} M_{2}$. Examples of this indexed product are shown in Figure 9.

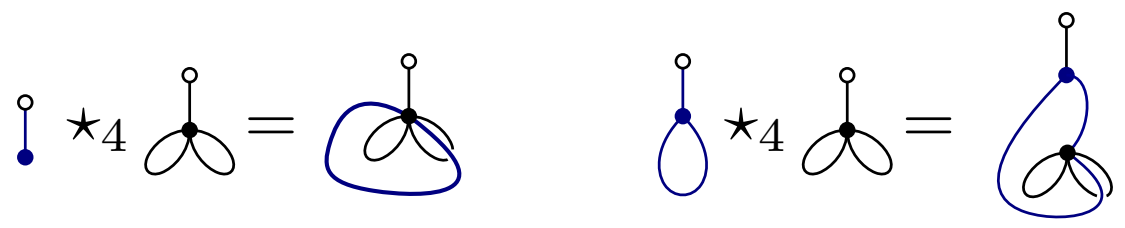

Figure 9: Illustration of the indexed product for bridgeless maps. 


\section{Description of the main bijections}

With all the tools we have introduced, it is now easy to construct explicit bijections between connected diagrams and bridgeless maps.

\subsection{Natural bijections}

We establish first a bijection between bridgeless maps and connected diagrams, which we denote $\theta$.

Definition 20 (Bijection $\boldsymbol{\theta}$ between bridgeless maps and connected diagrams). Let $M$ be a bridgeless map.

- If $M$ is reduced to a root, then $\theta(M)$ is the one-chord diagram.

- Otherwise, $M$ is of the form $M_{1} \star_{i} M_{2}$. Then $\theta(M)$ is equal to $\theta\left(M_{1}\right) \star_{i} \theta\left(M_{2}\right)$, where $\theta\left(M_{1}\right)$ and $\theta\left(M_{2}\right)$ are computed recursively.
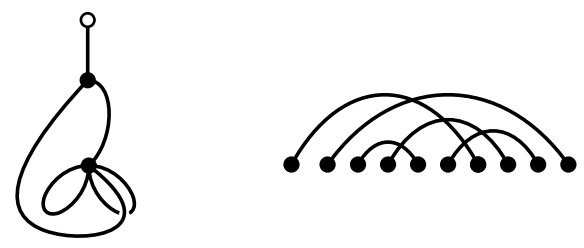

Figure 10: A bridgeless map and a connected diagram in bijection under $\theta$.

The mapping $\theta$ is provably bijective since we can define its inverse $\theta^{-1}$ by symmetry. Figure 10 presents a bridgeless map and a connected diagram in bijection under $\theta$, the decompositions of which are shown by Figures 4 and 8 .

As mentioned in the introduction, it was already known that rooted maps are in bijection with indecomposable diagrams [24, 25, 8]. However, this known bijection does not restrict to a bijection between bridgeless maps and connected diagrams, so we will now give one which does.

Definition 21 (Bijection $\phi$ between maps and indecomposable diagrams). Let $M$ be a combinatorial map. We define here the indecomposable diagram $\phi(M)$ as follows. (Figure 11 illustrates this definition.)

- If $M$ is reduced to the root, then $\phi(M)$ is the one-chord diagram.

- Assume that the root edge of $M$ is a bridge, i.e. $M$ is of the form $\operatorname{MapIns}_{M_{\downarrow}, 1}\left(M_{\uparrow}\right)$ for some $M_{\downarrow}$ and $M_{\uparrow}$. Then $\phi(M)$ is defined as

$$
\phi(M)=\operatorname{DiagIns}_{\phi\left(M_{\downarrow}\right), 1}\left(\phi\left(M_{\uparrow}\right)\right) .
$$

(The diagrams $\phi\left(M_{\downarrow}\right)$ and $\phi\left(M_{\uparrow}\right)$ are defined recursively.) 
- Assume that the root edge of $M$ is not a bridge, i.e. $M$ is of the form $M=$ $\operatorname{RootIns}_{k}\left(M^{\prime}\right)$. Then $\phi(M)$ is defined as

$$
\phi(M)=\operatorname{RootIns}_{k}\left(\phi\left(M^{\prime}\right)\right) .
$$

(The diagram $\phi\left(M^{\prime}\right)$ is defined recursively.)
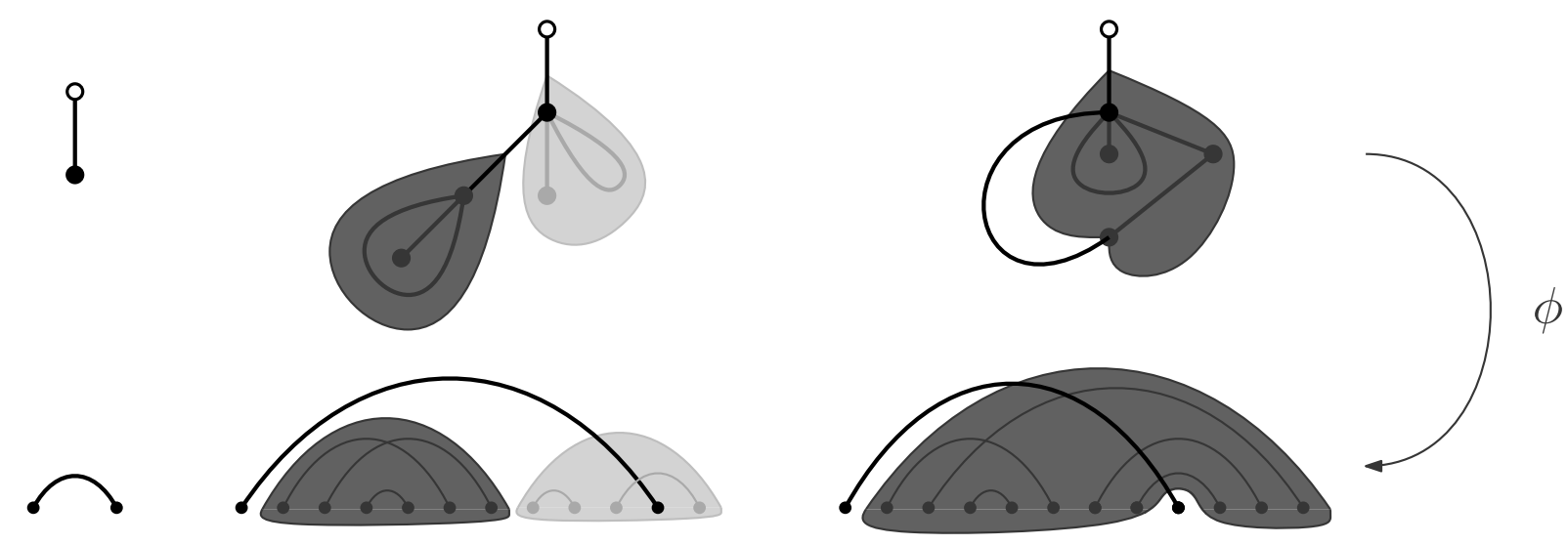

Figure 11: How $\phi$ is defined.

Remarkably, the two previous bijections are compatible with each other.

Theorem 22. The bijection $\phi$ is a bijection between rooted maps and indecomposable diagrams whose restriction to bridgeless maps is $\theta$. (Therefore, $\phi$ sends bridgeless maps to connected diagrams.)

The proof will be postponed for the next subsection.

\subsection{Extension of $\theta$ and equality between bijections}

In this subsection, we give another description of $\phi$, which is directly based on $\theta$. To do so, we again exploit the fact that rooted maps and indecomposable diagrams have equivalent decompositions, but now in terms of bridgeless maps and connected diagrams. The next proposition states those decompositions for both families, the principle of which is illustrated in Figure 12.

Proposition 23. Decomposition of diagrams. Any indecomposable diagram $D$ can be uniquely decomposed as a connected diagram $C$ and a sequence $\left(D_{1}, i_{1}\right), \ldots,\left(D_{k}, i_{k}\right)$ where each $D_{j}$ is an indecomposable diagram and $i_{j}$ is a integer such that $i_{1} \leqslant \cdots \leqslant i_{k}$ and

$$
D=\operatorname{DiagIns}_{D_{1}, i_{1}} \circ \operatorname{DiagIns}_{D_{2}, i_{2}} \circ \cdots \circ \operatorname{DiagIns}_{D_{k}, i_{k}}(C) .
$$

Decomposition of maps. Any map $M$ can be uniquely decomposed as a bridgeless map $M_{B}$ and a sequence $\left(M_{1}, i_{1}\right), \ldots,\left(M_{k}, i_{k}\right)$ where each $M_{j}$ is a map and $i_{j}$ is a integer such that $i_{1} \leqslant \cdots \leqslant i_{k}$ and

$$
M=\operatorname{MapIns}_{M_{1}, i_{1}} \circ \operatorname{MapIns}_{M_{2}, i_{2}} \circ \cdots \circ \operatorname{MapIns}_{M_{k}, i_{k}}\left(M_{B}\right) .
$$



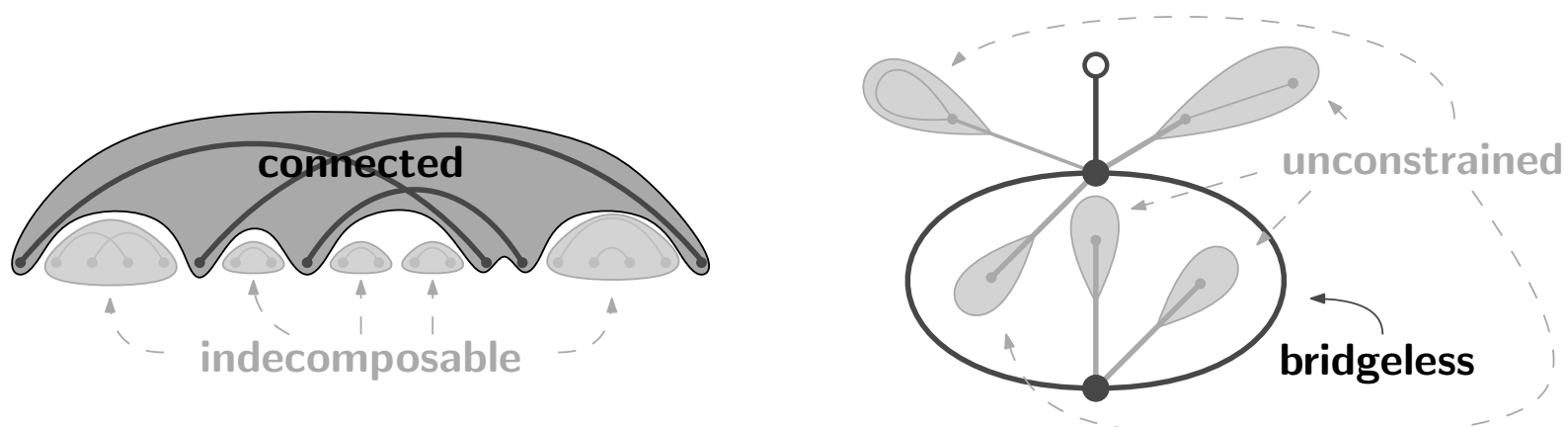

Figure 12: Left. Decomposition of an indecomposable diagram. Right. Decomposition of an unconstrained map. It is also the image of the diagram under $\phi=\bar{\theta}$.

Proof. Indecomposable diagrams. Here $C$ is the connected component of $D$ that includes the root chord. We can recover $D$ from $C$ by inserting in each interval of $C$ a sequence of indecomposable diagrams. We can do that starting from the right and ending to the left, which gives the above decomposition.

Maps. Here $M_{B}$ is the "bridgeless component" of the root (see right side of Figure 12). We recover $M$ from $M_{B}$ by grafting on each corner of $M_{B}$ a sequence of combinatorial maps. This can be done in the decreasing order for the Bridge First Labeling of $M_{B}$.

Definition 24 (Definition of $\overline{\boldsymbol{\theta}}$ ). Consider a map $M$. Let

$$
M=\operatorname{MapIns}_{M_{1}, i_{1}} \circ \operatorname{MapIns}_{M_{2}, i_{2}} \circ \cdots \circ \operatorname{MapIns}_{M_{k}, i_{k}}\left(M_{B}\right)
$$

be the decomposition of $M$ described by Proposition 23. Then $\bar{\theta}(M)$ is defined as the diagram

$$
\bar{\theta}(M)=\operatorname{DiagIns}_{\bar{\theta}\left(M_{1}\right), i_{1}} \circ \operatorname{DiagIns}_{\bar{\theta}\left(M_{2}\right), i_{2}} \circ \cdots \circ \operatorname{Diag}_{\operatorname{Ins}_{\bar{\theta}}\left(M_{k}\right), i_{k}}\left(\theta\left(M_{B}\right)\right) .
$$

where $\theta$ is the bijection defined by Definition 20 and where $\bar{\theta}\left(M_{1}\right), \ldots, \bar{\theta}\left(M_{k}\right)$ are computed recursively ${ }^{3}$.

It is easy to prove that $\bar{\theta}$ is a bijection since $\bar{\theta}^{-1}$ can be similarly defined by swapping the roles of maps and diagrams. Moreover, when $M$ is bridgeless, we have $k=0$. Therefore, the restriction of $\bar{\theta}$ to bridgeless maps is, by definition, equal to $\theta$.

Theorem 22 then results from the following proposition.

Proposition 25. We have $\phi=\bar{\theta}$.

\footnotetext{
${ }^{3}$ Since we have $\bar{\theta}=\theta$ for bridgeless diagrams, the base cases of the recursion are well treated.
} 
Proof. We prove that $\phi(M)=\bar{\theta}(M)$ for any map $M$ by induction on the size of the map. The base case (when $M$ reduced to a root) is given by the definitions.

Let $M$ be a map of size $>1$, which we decompose (by Proposition 23) as

$$
M=\operatorname{MapIns}_{M_{1}, i_{1}} \circ \operatorname{MapIns}_{M_{2}, i_{2}} \circ \cdots \circ \operatorname{MapIns}_{M_{k}, i_{k}}\left(M_{B}\right) .
$$

There are three possibilities.

1. The root edge of $\boldsymbol{M}$ is a bridge. Since the root edge of $M$ is a bridge, we have $i_{1}=1$. Moreover, referring to the notation of Definition 21, $M_{1}=M_{\downarrow}$ and $M_{\uparrow}=\operatorname{MapIns}_{M_{2}, i_{2}} \circ \cdots \circ \operatorname{MapIns}_{M_{k}, i_{k}}\left(M_{B}\right)$. By using twice the definition of $\bar{\theta}$, we have

$$
\begin{aligned}
\bar{\theta}(M) & =\operatorname{DiagIns}_{\bar{\theta}\left(M_{1}\right), 1} \circ \operatorname{DiagIns}_{\bar{\theta}\left(M_{2}\right), i_{2}} \circ \cdots \circ \operatorname{DiagIns}_{\bar{\theta}\left(M_{k}\right), i_{k}}\left(\theta\left(M_{B}\right)\right) \\
& =\operatorname{DiagIns}_{\bar{\theta}\left(M_{1}\right), 1}\left(\bar{\theta}\left(M_{\uparrow}\right)\right) .
\end{aligned}
$$

But by induction, $\bar{\theta}\left(M_{1}\right)=\phi\left(M_{1}\right)$ and $\bar{\theta}\left(M_{\uparrow}\right)=\phi\left(M_{\uparrow}\right)$. Thus, we recover the definition of $\phi$, and so $\bar{\theta}(M)=\phi(M)$.

2. The root edge of $M$ is not a bridge and its deletion in $M_{B}$ gives a bridgeless map $\boldsymbol{M}_{B}^{\prime}$. Then $M_{B}$ is of the form $M_{B}=\operatorname{RootIns}_{i}\left(M_{B}^{\prime}\right)$. By definition of $\theta$, we have $\theta\left(M_{B}\right)=\operatorname{RootIns}_{i}\left(\theta\left(M_{B}^{\prime}\right)\right)$. Therefore

$$
\bar{\theta}(M)=\operatorname{DiagIns}_{\bar{\theta}\left(M_{1}\right), i_{1}} \circ \cdots \circ \operatorname{DiagIns}_{\bar{\theta}\left(M_{k}\right), i_{k}} \circ \operatorname{RootIns}_{i}\left(\theta\left(M_{B}^{\prime}\right)\right) .
$$

Since $i_{1}>1$, we can use Lemma 18 to slide the operation RootIns to the left:

$$
\bar{\theta}(M)=\operatorname{RootIns}_{j} \circ \operatorname{DiagIns}_{\bar{\theta}\left(M_{1}\right), j_{1}} \circ \cdots \circ \operatorname{DiagIns}_{\bar{\theta}\left(M_{k}\right), j_{k}}\left(\theta\left(M_{B}^{\prime}\right)\right) .
$$

(The integers $j, j_{1}, \ldots, j_{k}$ are given by Lemma 18.) But by Lemma 15 we also have

$$
\begin{aligned}
M & =\operatorname{MapIns}_{M_{1}, i_{1}} \circ \cdots \circ \operatorname{MapIns}_{M_{k}, i_{k}} \circ \operatorname{RootIns}_{i}\left(M_{B}^{\prime}\right) \\
& =\operatorname{RootIns}_{j} \circ \operatorname{MapIns}_{M_{1}, j_{1}} \circ \cdots \circ \operatorname{MapIns}_{M_{k}, j_{k}}\left(M_{B}^{\prime}\right),
\end{aligned}
$$

with the same $j, j_{1}, \ldots, j_{k}$ as above. So using successively the definition of $\phi$, the induction hypothesis, and the definition of $\bar{\theta}$,

$$
\begin{aligned}
\phi(M) & =\operatorname{RootIns}_{j}\left(\phi\left(\operatorname{MapIns}_{M_{1}, j_{1}} \circ \cdots \circ \operatorname{MapIns}_{M_{k}, j_{k}}\left(M_{B}^{\prime}\right)\right)\right) \\
& =\operatorname{RootIns}_{j}\left(\bar{\theta}\left(\operatorname{MapIns}_{M_{1}, j_{1}} \circ \cdots \circ \operatorname{MapIns}_{M_{k}, j_{k}}\left(M_{B}^{\prime}\right)\right)\right) \\
& =\operatorname{RootIns}_{j}\left(\operatorname{DiagIns}_{\bar{\theta}\left(M_{1}\right), j_{1}} \circ \cdots \circ \operatorname{DiagIns}_{\bar{\theta}\left(M_{k}\right), j_{k}}\left(\theta\left(M_{B}^{\prime}\right)\right)\right)=\bar{\theta}(M) .
\end{aligned}
$$

3. The root edge of $M$ is not a bridge and its deletion in $M_{B}$ does not give a bridgeless map. Since the deletion of the root edge of $M_{B}$ does not give a bridgeless map, $M_{B}$ is an indexed product (see Definition 19) of the form

$$
M_{B}=M^{\prime} \star_{\ell} M^{\prime \prime}
$$


where $M^{\prime}$ is a bridgeless map of size $>1$, and $M^{\prime \prime}$ is some bridgeless map. Since $M^{\prime}$ has size more than 1 , it can be put in the form $\operatorname{RootIns}_{i}(\widehat{M})$. Then, by definition of the indexed product, $M_{B}$ can be written as

$$
M_{B}=\operatorname{RootIns}_{i+\ell} \circ \operatorname{MapIns}_{M^{\prime \prime}, i}(\widehat{M}) .
$$

Since by definition, $\theta\left(M_{B}\right)=\theta\left(M^{\prime}\right) \star_{\ell} \theta\left(M^{\prime \prime}\right)$, we also have

$$
\theta\left(M_{B}\right)=\operatorname{RootIns}_{i+\ell} \circ \operatorname{DiagIns}_{\theta\left(M^{\prime \prime}\right), i}(\theta(\widehat{M})) .
$$

Then, using the same techniques as the previous case, we apply the definition of $\bar{\theta}$ :

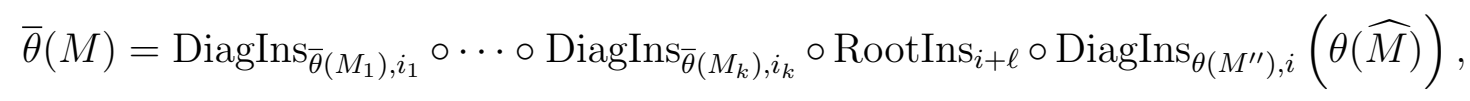

we commute the operators thanks to Lemma 15:

$$
\bar{\theta}(M)=\operatorname{RootIns}_{j} \circ \operatorname{DiagIns}_{\bar{\theta}\left(M_{1}\right), j_{1}} \circ \cdots \circ \operatorname{DiagIns}_{\bar{\theta}\left(M_{k}\right), j_{k}} \circ \operatorname{DiagIns}_{\theta\left(M^{\prime \prime}\right), i}(\theta(\widehat{M})),
$$

we recognize the definition of $\bar{\theta}$ :

$$
\bar{\theta}(M)=\operatorname{RootIns}_{j}\left(\bar{\theta}\left(\operatorname{MapIns}_{M_{1}, j_{1}} \circ \cdots \circ \operatorname{MapIns}_{M_{k}, j_{k}} \circ \operatorname{MapIns}_{M^{\prime \prime}, i}(\widehat{M})\right)\right),
$$

and we use the induction hypothesis and the definition of $\phi$ to conclude.

\subsection{Planar maps as diagrams with forbidden patterns}

Planarity of a combinatorial map can be recognized using its Euler characteristic.

Definition 26 (Faces, Euler characteristic, planarity). Let $M=(H, \sigma, \alpha, r)$ be a rooted combinatorial map (potentially with dangling edges). The faces of $M$ are the orbits of the composite permutation $\sigma \alpha$. The root face is the face containing $r$. The Euler characteristic of $M$ is defined by

$$
\chi(M)=|\operatorname{orbit}(\sigma)|+|\operatorname{orbit}(\alpha)|+|\operatorname{orbit}(\sigma \alpha)|-|H| .
$$

$M$ is said to be planar if $\chi(M)=2$.

We here characterize the image of planar maps under the previous bijections.

Proposition 27. Under $\phi$ planar rooted maps with $n$ edges are in bijection with indecomposable diagrams with $n$ chords which do not contain the configuration of Figure 13 as a subdiagram. Thus, restricting to $\theta$, a bridgeless map is planar if and only if the corresponding connected diagram does not contain the forbidden configuration.

Before we prove this result we need a couple more definitions. 


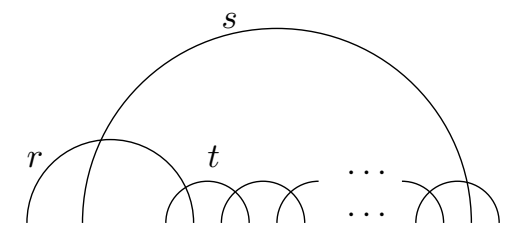

Figure 13: Forbidden configuration for diagrams corresponding to planar maps.

Definition 28 (Internal/external corners). Given a planar rooted map $M$, a corner whose second component is contained in the root face is called an external corner of $M$. A corner which is not external is called internal.

Definition 29 (Blocked/unblocked intervals). Given an indecomposable diagram $D$, an interval in $D$ is a blocked interval if it is

- under the root chord and under at least one other chord in the same connected component as the root chord,

- or already blocked in a component of the diagram obtained by removing the root chord.

An interval which is not blocked is called unblocked.

Lemma 30. Let $M$ be a planar rooted map. The blocked intervals of $\phi(M)$ correspond to the internal corners of $M$.

Proof. We prove this lemma by induction. In the base case $M$ is the trivial map, which has no internal corners, corresponding to the one-chord diagram with no blocked intervals. Suppose $M$ is a planar map with more than one half-edge. There are two cases.

Suppose the root edge of $M$ is a bridge, so that $M=\operatorname{MapIns}_{M_{1}, 1}\left(M_{2}\right)$, where $M_{1}$ and $M_{2}$ are planar maps. All of the internal corners of $M_{1}$ and $M_{2}$ remain internal in $M$, and all of the external corners remain external (the external root corner of $M_{2}$ splits into two external corners in $M)$. Likewise, since the connected components remain the same, all of the blocked intervals of $\phi\left(M_{1}\right)$ and $\phi\left(M_{2}\right)$ remain blocked in $\phi(M)=\operatorname{DiagIns}_{\phi\left(M_{1}\right), 1}\left(\phi\left(M_{2}\right)\right)$, and unblocked intervals remain unblocked. By induction, the internal corners of $M_{1}$ and $M_{2}$ correspond to the blocked corners of $\phi\left(M_{1}\right)$ and $\phi\left(M_{2}\right)$, so this ends the proof.

The other case is $M=\operatorname{RootIns}_{k}\left(M_{1}\right)$, where $M_{1}$ is planar and $k$ is an external corner of $M_{1}$. The external corners of $M$ are its root corner, along with the external corners of $M_{1}$ which counterclockwisely follow the corner labeled by $k$. Expressed in terms of the Bridge First Labeling of $M_{1}$, these external corners are those with an index larger than $k$, which correspond to the corners with index $\geqslant k+2$ in $M$. On the other hand, for the diagram $\phi(M)=\operatorname{RootIns}_{k}\left(\phi\left(M_{1}\right)\right)$, the new root chord blocks the intervals 2 through $k+1$ in $\phi(M)$, while leaving the other intervals unchanged. By induction, internal corners of $M_{1}$ correspond to blocked corners of $\phi\left(M_{1}\right)$, so this concludes the proof. 
Proof of Proposition 27. Let us first observe that $\operatorname{MapIns}_{M_{1}, k}\left(M_{2}\right)$ is planar if and only if both $M_{1}$ and $M_{2}$ are planar, while $\operatorname{RootIns}_{k}\left(M_{1}\right)$ is planar if and only if $M_{1}$ is planar and $k$ is an external corner of $M_{1}$.

Now, consider a map $M$ as built iteratively according to the induction used in the definition of $\phi(M)$.

Suppose $M$ is nonplanar. Then at some stage in this construction we must have built a map $\operatorname{RootIns}_{k}\left(M_{1}\right)$ by inserting a new root edge into an internal corner of a planar map $M_{1}$. Let $M^{\prime}=\operatorname{RootIns}_{k}\left(M_{1}\right)$. We will now proceed to show that $\phi\left(M^{\prime}\right)$ has the configuration of Figure 13.

By Lemma 30, since RootIns ${ }_{k}\left(M_{1}\right)$ comes from the insertion of a new root edge into an internal corner, $\operatorname{RootIns}_{k}\left(\phi\left(M_{1}\right)\right)$ comes from the insertion of a a new root into a blocked interval $k$ of $\phi\left(M_{1}\right)$. Let $r$ be the root chord of $\phi\left(M^{\prime}\right)=\operatorname{RootIns}_{k}\left(\phi\left(M_{1}\right)\right)$. Since the interval where $r$ is inserted is blocked, there is some subdiagram of $\phi\left(M_{1}\right)$ where the first point of the definition of blocked interval holds. In other words, there is a connected subdiagram $C$ of $\phi\left(M_{1}\right)$ with root chord $s$, and when $r$ is inserted via $\operatorname{RootIns}_{k}\left(\phi\left(M_{1}\right)\right)$, then $r$ crosses both $s$ and another chord $t$ of $C$. Since $s$ is the root of $C$, other chords of $C$ can only cross $s$ on the right. Also $C$ is connected, so there is a chain of chords connecting $t$ to the right hand side of $s$. By taking a minimum chain we can guarantee that the chords in the chain go from left to right and do not cross chords which are not their immediate neighbors. Thus $r, s$ and the chain give the forbidden configuration in Figure 13.

Further operations of RootIns and DiagIns preserve the forbidden configuration, and so $\phi(M)$ also has the forbidden configuration. Thus we have proved that if $M$ is nonplanar then $\phi(M)$ has the forbidden configuration.

Now consider the converse. With no planarity assumption on $M$, suppose that $\phi(M)$ has the forbidden configuration. Then at some stage in the construction of $\phi(M)$ we must have built a diagram $\operatorname{RootIns}_{k}\left(\phi\left(M_{1}\right)\right)$ so that the newly constructed root chord, call it $r$, crosses the root chord $s$ of $\phi\left(M_{1}\right)$ and another chord $t$ of $\phi\left(M_{1}\right)$ and there is a chain in $\phi\left(M_{1}\right)$ joining the right end points of $t$ and $s$. In particular the $k$ th interval of $\phi\left(M_{1}\right)$ is under $s$ and $t$, both of which are in the same connected component of $\phi\left(M_{1}\right)$. Thus this interval is blocked in $\phi\left(M_{1}\right)$. By Lemma 30, it must correspond to an internal corner of $M_{1}$, and so $\operatorname{RootIns}_{k}\left(M_{1}\right)$ is nonplanar. Further, once the map becomes nonplanar, no sequence of RootIns or MapIns operations can make the map planar again, and so $M$ is also nonplanar.

An interesting question (posed by a referee) is whether indecomposable diagrams corresponding to genus 1 maps can be similarly characterized by a list of forbidden configurations. We leave this as an open problem.

\section{New perspectives on chord diagram expansions in QFT}

Interestingly, by the work of some of the authors with other collaborators $[22,15,10]$, rooted connected chord diagrams appear in quantum field theory where they give series 
solutions to certain Dyson-Schwinger equations. We are going to see that the $\theta$ bijection of Section 4 will simplify some formulas in this theory: Corollary 48 recasts the main result of [15] in map language; Table 3 shows how important parameters translate, some becoming considerably more natural; and along the way we prove and generalize a conjecture of Hihn (see the discussion at the end of Subsection 5.4). This section will begin with some of the physics context. The reader who simply wants to get to the combinatorics can jump to Definition 31 in Subsection 5.2 and then also skip the discussion after (10), returning at (13).

\subsection{Context: Feynman graphs and Dyson-Schwinger equations}

This physics work lies within the field of perturbative quantum field theory, which is the approach of studying quantum field theory by expanding in (hopefully small) parameters. One well-established and still very useful way to do this is by expanding in Feynman graphs (also known as Feynman diagrams).

Feynman graphs are graphs with edge types corresponding to the different particles in the quantum field theory in question and with vertex types corresponding to the possible interactions in the theory. Feynman diagrams may also have external edges, which are best thought of as half-edges which are not paired into an edge with another half-edge, analogous to the root in our combinatorial maps. For example the Feynman graphs in Figure 14 is in a theory (Yukawa theory) with two edge types, an unoriented edge shown with a dashed line and an oriented edge. This theory has one vertex type with one ingoing and one outgoing oriented edge and one dashed edge. The example in the figure has two external edges (on the right and left). The size of a Feynman graph is usually taken to be the dimension of its cycle space, so one and four respectively for the two example graphs in Figure 14; this is called the loop number in the physics literature. For formal definitions in a compatible language see sections 5.1 and 5.2 of [32]. In perturbative quantum field theory each Feynman graph is associated to an integral called the Feynman integral, where each edge and vertex of the graph contributes a factor to the integrand (or more generally a factor to each term in a sum giving the integrand). A few examples can be found in section 5.6 of [32] and many more along with more information on where these integrals come from can be found in any quantum field theory textbook, for example section 6.1 of [17].

Summing Feynman integrals corresponding to Feynman graphs with the same multiset of external edges computes the scattering amplitude for the physical process where the external edges are the particles coming into and going out of the process, as for example in a particle accelerator where some known particles are collided and the output particles of the collision are measured by the detectors. As in enumerative combinatorics we can restrict to considering connected Feynman graphs by taking a logarithm and to bridgeless Feynman graphs by taking a Legendre transform [18]. The resulting sums of Feynman integrals over all connected bridgeless Feynman graphs with a given multiset of external edges are Green functions of the theory. These sums are still very difficult to analyze, which motivates to study special cases where we put further restrictions on the graphs. We will also call these more restricted sums Green functions. 
Dyson-Schwinger equations are the quantum analogues of the classical equations of motion. The solutions of such functional equations are the same Green functions of the quantum field theory discussed above. It turns out that these equations have a nice underlying combinatorial aspect. They capture the decomposition of Feynman graphs into subgraphs, so viewing perturbative expansions as intricately weighted generating functions, the Dyson-Schwinger equations can be interpreted as equations for the generating functions of appropriate combinatorial classes of Feynman diagrams. Furthermore these functional equations mirror the combinatorial decomposition of the graphs. Using the universal property of the so-called Connes-Kreimer Hopf algebra of rooted trees, we can also view the Dyson-Schwinger equations as functional equations for classes of rooted trees. This happens by using the rooted trees to represent insertion structures of Feynman diagrams.

Dyson-Schwinger equations can be expressed in a number of different forms which are not obviously related. The first form one might find is an equation involving the derivative of an important object known as the action in terms of another function of the field. The precise definitions would be too much of a digression for the present purposes, but with a similar argument to the derivation of the Feynman integrals themselves, Dyson-Schwinger equations in this form can be converted into diagrammatic form, namely, in terms of sums over Feynman graphs. One fairly concrete presentation of this material can be found in sections V.A and V.B of the notes [28].

The Dyson-Schwinger equations in diagrammatic form are essentially combinatorial specifications for classes of Feynman graphs interpreted at the level of the Green functions. A good concrete example is the special case of bridgeless connected Yukawa theory graphs formed by iteratively inserting the left hand graph of Figure 14 into itself. Diagrammatically this Dyson-Schwinger equation is often written

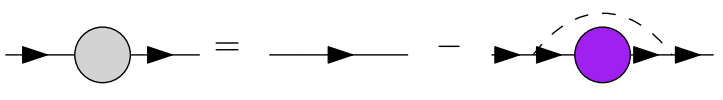

where a graph with a blob represents a sum of Feynman integrals, running over all possible graphs that can be formed by substituting some graph into the blob (while staying compatible with the edge and vertex types allowed in the theory). Furthermore, for the grey (lighter) blob we restrict to connected bridgeless graphs, while for the purple (darker) blob a sequence of any number of connected bridgeless graphs can be inserted. This is usually written

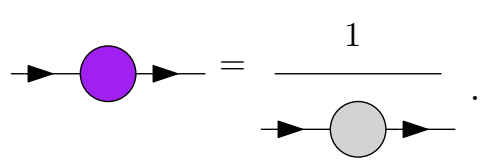

To interpret this second equation, note that the previous equation for the graph with the grey (lighter) blob told us that the sum corresponding to the graph with the grey blob begins with just a single edge (known as a propagator since it corresponds to a particle propagating without interacting). Inserting a single edge into an edge is the identity operation (because there are no vertices at the ends, so we do not create subdivisions), and so we can treat it as 1 . Thus the graph with the grey blob corresponds to an invertible 


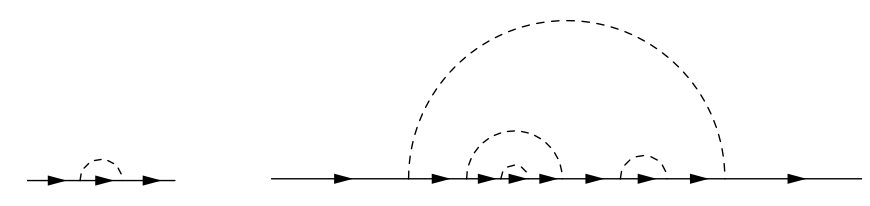

Figure 14: Two examples of Feynman graphs which occurs in the simpler Dyson-Schwinger equation.

series and in particular the inverse is the geometric series of the second term on the right of (7). An example of two Feynman graphs appearing in this expansion are given in Figure 14.

Translated into more combinatorial terms, these equations are telling us that the class of graphs we are building consists of an outer arc and inside a sequence of smaller graphs from the class; that is we have a graph class in bijection with rooted trees with the classical combinatorial specification $\mathcal{T}=\bullet \times \operatorname{SEQ}(\mathcal{T})$ and corresponding generating function equation $T(x)=x /(1-T(x))$. The Dyson-Schwinger equation rewrites this in terms of $U(x)=1-T(x)$ and so the generating function equation becomes $U(x)=$ $1-x / U(x)$. The only difference between this last generating function equation and (7) is that rather than an ordinary generating function we have the Green function, so we are weighting each graph with its Feynman integral rather than just counting them in the sum.

Analogously to how the combinatorial specification converts to the functional equation for the generating function, the diagrammatic form of the Dyson-Schwinger equation can be converted to an analytic form: an integral equation which arises when each Feynman graph is replaced by its Feynman integral. The particular example we have been using was studied in this integral form and solved by Broadhurst and Kreimer in [6]. It can also be found in notation closer to what we are using here as a running example in [31] or [30] (see particularly example 3.5), or in chapter 6 of [32].

However, this is still not the form we want for the Dyson-Schwinger equation. Example 3.7 of [31] of [30] shows how to convert this integral form of the Dyson-Schwinger equation into a differential form. In this example the equation becomes

$$
G(x, L)=\left[\rho^{0}\right]\left(1-x G\left(x, \frac{d}{d(-\rho)}\right)^{-1}\left(e^{-L \rho}-1\right) F(\rho)\right)
$$

where

$$
F(\rho)=f_{0} \rho^{-1}+f_{1}+f_{2} \rho+\cdots
$$

is the series expansion of the regularized Feynman integral of the primitive Feynman graph (see [22] for details). Note that $e^{-L \rho}-1$ has no constant as a series in $\rho$, so $\left(e^{-L \rho}-1\right) F(\rho)$ is in fact a power series, not a Laurent series, in $\rho$.

There are a number of observations to be made about this equation. First of all Equation (8) should be interpreted as a formal series equation. The unknown is the bivariate series $G(x, L)$. The appearance of the differential operator $d / d(-\rho)$ in $G$ should 
be interpreted by substituting $d / d(-\rho)$ for $L$ in the formal power series $G(x, L)^{-1}$ and then viewing this term by term as a formal differential operator acting on $\left(e^{-L \rho}-1\right) F(\rho)$.

To continue the Yukawa example, in that case we have $F(\rho)=1 /(\rho(1-\rho))$ and so then $G(x, L)$ in $(8)$ is the sum indexed by all Feynman graph generated by $(7)$ where each graph contributes its Feynman integral. The variable $L$ is defined as $L=\log \left(q^{2} / \mu^{2}\right)$ where $q$ is the momentum coming in and going out of each graph and $\mu$ is a renormalization constant, and $x$ is the coupling constant (giving the strength of the interaction).

If we took a different class of Feynman graphs, potentially in a different quantum field theory, but with the same combinatorics, then the only thing that would change in (8) would be $F(\rho)$. For example, quantum electrodynamics is the quantum theory of photons and electrons (and positrons), and also has one directed edge type (corresponding to electrons and positrons) and one undirected edge type (corresponding to photons) and the only kind of vertex is the one with one photon edge along with an incoming and an outgoing directed edge. The graphs combinatorics is the same and so the analogous class of graphs with photon edges (usually drawn with wiggly lines) replacing the dashed edges would also satisfy $(8)$ but $F(\rho)$ would be a different series.

\subsection{Context: chord diagram expansions of Dyson-Schwinger equations}

One of us has a program with various collaborators to better understand the combinatorial underpinnings of Dyson-Schwinger equations. One of the successes of this program has been to solve certain classes of Dyson-Schwinger equations using expansions indexed by chord diagrams, first in [22] and then generalized in [15].

The paper [22] considered the Dyson-Schwinger equation (8) discussed above. This corresponds to the situation where one primitive ${ }^{4}$ Feynman graph is inserted into itself in all possible ways on one internal edge.

The paper [15] considers more general Dyson-Schwinger equations, see Equation (10), though they are still not the most general possible. The form studied in [15] captures classes of graphs which can be made from inserting more than one different graph into itself, and so in particular the graphs may be nonplanar. The parameter $s$ in (10) lets the equation also capture different underlying combinatorics of edge and vertex types. More details on $s$ are given below where (10) is discussed.

Two things are missing before we can consider that we have captured all DysonSchwinger equations from quantum field theory. First, the $F_{i}$ should be multivariate. This corresponds to the fact the momentum of different particles in the process is in general different. Second, we should work with systems of Dyson-Schwinger equations, as we are not interested in just one multiset of external edges, but with many, and we are interested in how the different external edge structures interact. Some of this is work in progress with one of us and a student; the program of understanding Dyson-Schwinger equations by chord diagram expansions is ongoing.

\footnotetext{
${ }^{4}$ In this case primitive means primitive in the renormalization Hopf algebra, or equivalently having no proper subdivergences.
} 


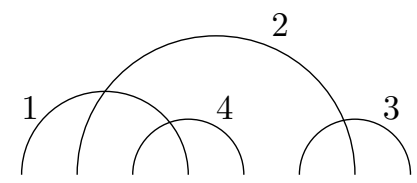

Figure 15: An example where the intersection order (indicated) is not the order by first end point.

To proceed, we need two further definitions concerning rooted connected chord diagrams which arise from the quantum field theory application, see $[22,15]$.

Definition 31 (Intersection order). The intersection order of the chords of a rooted connected diagram $C$ is defined as follows.

- The root chord of $C$ is the first chord in the intersection order.

- Remove the root chord of $C$ and let $C_{1}, C_{2}, \ldots, C_{k}$ be the connected components of the result ordered by their first vertex.

- For the intersection order of $C$, after the root chord come all the chords of $C_{1}$ ordered recursively in the intersection order, then all the chords of $C_{2}$ ordered by intersection order, and so on.

This intersection order is not in general the same as the order by first endpoint, see Figure 15 for an example. The intersection order and the order by first endpoint both define total orders on chords extending the partial order induced by paths in the intersection graph (recall Definition 3).

Definition 32 (Terminal chord). A chord $c$ is terminal if the left endpoint of every chord intersecting $c$ is to the left of $c$.

Equivalently, a chord $c$ is terminal if it does not cross any chords larger than it in the intersection order; or (third equivalent definition) a chord is terminal if it is a sink in the intersection graph. For example, in Figure 15, only chords labeled by 3 and 4 are terminal.

The main result of [22] was to solve the Dyson-Schwinger equation (8) as

$$
G(x, L)=1-\sum_{C}\left(\sum_{i=1}^{b(C)} f_{b(C)-i} \frac{(-L)^{i}}{i !}\right) x^{|C|} f_{0}^{|C|-\ell} \prod_{j=2}^{\ell} f_{t_{j}-t_{j-1}}
$$

where the sum is over connected diagrams $C$ and the terminal chords of $C$ are indexed by $b(C)=t_{1}<t_{2}<\cdots<t_{\ell}$ in the intersection order. Note that this gives $G(x, L)-1$ as a kind of strangely weighted generating function of connected diagrams. Its first terms are given by

$$
G(x, L)-1=f_{0} L x+\left(f_{1} L-f_{0} \frac{L^{2}}{2}\right) f_{0} x^{2}+\cdots,
$$


which respectively correspond to the one-chord diagram and the connected two-chords diagram. In [10] two of us used tools of asymptotic combinatorics to better understand some of these parameters and in particular were able to conclude that in each of the nextto $^{k}$-leading log expansions only $f_{0}$ and $f_{1}$ contribute. This was subsequently generalized to the case of (10) in [9].

We can compare (9) to the original Feynman graph expansion. Both are expansions over combinatorial objects yielding the same series $G(x, L)$. In the Feynman graph expansion each graph has a very complicated contribution, namely its Feynman integral, to the sum. Thus if we want to find properties like the asymptotic behavior of $G(x, L)$, the Feynman graph expansion hides important features in the Feynman integrals and so only a combinatorial analysis can get us so far.

In the chord diagram expansion each chord diagram has a simple contribution to the sum - just certain monomials in the $f_{i}$. This means that, in principle, combinatorial tools could fully understand $G(x, L)$, and in practice we can make good progress as in [10]. On the other hand, we have lost a physical interpretation for each diagram (the Feynman graphs directly represent particles and their interactions); each chord diagram just represents some terms in expansions of some Feynman graphs.

In [15], generalizing [22], one of us with Markus Hihn solves the Dyson-Schwinger equation

$$
G(x, L)=\left[\rho^{0}\right]\left(1-\sum_{k \geqslant 1} x^{k} G\left(x, \frac{d}{d(-\rho)}\right)^{1-s k}\left(e^{-L \rho}-1\right) F_{k}(\rho)\right)
$$

where $F_{k}(\rho)=\sum_{i \geqslant 0} a_{k, i} \rho^{i-1}$ and $s$ is a positive integer parameter. This Dyson-Schwinger equation corresponds to the case where we are still restricted to propagator corrections but now we can have any number of primitive Feynman graphs (the integer $k$ refers to their possible sizes, where the size is the dimension of the cycle space of the graph), and the number of insertion places is one less than $s$ times the size of the graph, where $s$ can be any positive integer.

Let us look at some more Feynman graphs in order to get a better feel for what these parameters mean. In the previous Yukawa example we were building Feynman graphs by inserting

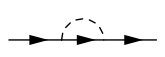

into itself in all possible ways. Another Feynman graph in this theory and with the same external edges but which can't be built out of insertions of the first is

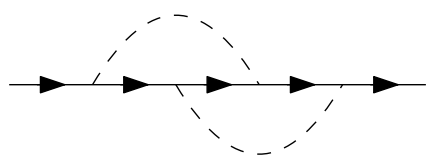

Notice that the first Feynman graph has one internal edge into which it can be inserted while the second has three. Any way of making another Feynman diagram with one more cycle but with the same edge and vertex types and the same external edges would have 

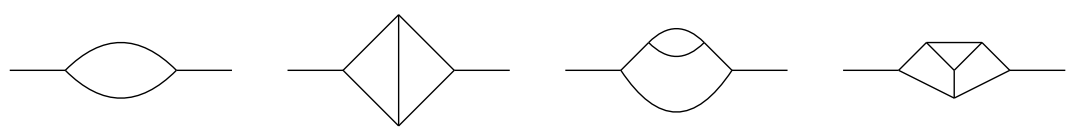

Figure 16: Some Feynman graphs in $\phi^{3}$.

five places to insert itself. This increases by two at each step corresponds to the fact that $s=2$. In a different quantum field theory, say scalar $\phi^{3}$ theory, the growth in insertion places would be different. Figure 16 shows some Feynman graphs in $\phi^{3}$ theory with two external edges. Note that number of insertion places increases by three as the dimension of the cycle space increases by one. This means that $s=3$ in this theory.

For formal defintions of insertion and related notions see section 2.2.3 of [30] or [31] and for more on counting insertion places see Proposition 3.9 of [30] of [31].

The main result of [15] is that (10) is solved by

$$
G(x, L)=1-\sum_{C}\left(\sum_{i=1}^{b(C)} a_{d(b(C)), b(C)-i} \frac{(-L)^{i}}{i !}\right) w(C) A(C) x^{\|C\|},
$$

where the first sum runs over all connected diagrams $C$, carrying a positive integer weight $d(c)$ on each of its chords $c$, and such that the position of the first terminal chord is $b(C)$. As for the other parameters, $|C|$ denotes the number of chords; $\|C\|$ is the sum of the chord weights; $t_{1}=b(C)<t_{2}<\cdots<t_{\ell}=|C|$ lists the positions of all the terminal chords in intersection order;

$$
w(C)=\prod_{m=1}^{|C|}\left(\begin{array}{c}
d(m) s+\nu(m)-2 \\
\nu(m)
\end{array}\right) ;
$$

and

$$
A(C)=\prod_{\substack{c \text { not } \\ \text { terminal }}} a_{d(c), 0} \prod_{j=2}^{\ell} a_{d\left(t_{j}\right), t_{j}-t_{j-1}} .
$$

For the definition of $w(C)$, we need another parameter $\nu(c)$ which is discussed in the next subsection. Note that again $G(x, L)-1$ is a weighted generating function of connected diagrams.

Example 33. As an example, take the diagram in Figure 15. Note that the terminal chords are chords 3 and 4 , so $b(C)=3$. If all the chords are weighted by 1 then $A(C)=$ $a_{1,0}^{2} a_{1,1}$. If the first chord is weighted by 2 while the rest are weighted by 1 then $A(C)=$ $a_{1,0} a_{2,0} a_{1,1}$, while if the fourth chord is weighted by 2 and the rest are weighted by 1 then $A(C)=a_{1,0}^{2} a_{2,1}$. Note that the weight of the first terminal chord does not affect $A(C)$.

Continuing the example, note that if all chords are weighted by $2 / s$ (since $s$ is an integer and the weights are nonnegative integers, this means $s=2$ and all weights are 1 or $s=1$ and all weights are 2) then $w(C)$ is independent of $\nu$ and equals 1 for all $C$. More 
generally, $\nu(C)$ will be defined in the next subsection, but for now taking it as given that $\nu(1)=\nu(2)=0$ and $\nu(3)=2$ and $\nu(4)=1$ then we can compute $w(C)$. Say $s=2$ and all chords are weighted by 1 except the third which has weight 2 , then $w(C)=\left(\begin{array}{l}3 \\ 2\end{array}\right)=3$. With the same weights but $s=3$ we get $w(C)=\left(\begin{array}{l}1 \\ 0\end{array}\right)\left(\begin{array}{l}1 \\ 0\end{array}\right)\left(\begin{array}{l}3 \\ 2\end{array}\right)\left(\begin{array}{l}7 \\ 6\end{array}\right)=21$.

The theorem stating that (13) solves (10) was shown by checking that the coefficients of the Dyson-Schwinger equation and the eventual solution both satisfy the same recurrences with the same initial conditions. This was done in two steps. First viewing each as a series in $L$ with coefficients which are functions of $\alpha$, these coefficients were shown to satisfy the same recurrence. For the Dyson-Schwinger equation this $L$-recurrence is the renormalization group equation, an important equation for quantum field theories.

The second step was to check that the linear coefficient in $L$ matches in the chord diagram expansion and the Dyson-Schwinger equation giving the initial conditions for the $L$-recurrence. These coefficients are themselves series in $x$ and the proof is again done by matching recurrences. However, this time the recurrence is more obscure, corresponding neither to a straightforward combinatorial decomposition nor to a standard physics identity. Stated as an identity of weighted generating functions this equation becomes what will be numbered by (16) in the next subsection. In [22] and [15] we understood this formula by passing to a class of rooted trees but this class was messy and we were not able to understand the formula directly on the chord diagrams. We will discuss this formula further, reinterpreting it in terms of rooted maps, and providing a combinatorial interpretation also at the level of rooted maps. This will show that the connection between chord diagrams and rooted maps can improve our understanding as the whole story can be formulated with one class of objects, namely rooted maps.

\subsection{Diagram parameters and binary trees}

To see how the bijection $\theta$ from connected diagrams to bridgeless maps helps simplify the situation, we need to understand these additional parameters as they were originally defined.

The first thing we need is a variant of the indexed product (see Figure 17 for an illustration).

Definition 34 (Variant product for connected diagrams). Let $C_{1}$ and $C_{2}$ be two connected diagrams and $i$ an integer between 1 and $2\left|C_{2}\right|-1$. The connected diagram $C_{1} \oplus_{i} C_{2}$ is defined as

$$
\begin{gathered}
\operatorname{RootIns}_{i}\left(C_{2}\right) \text { if } C_{1} \text { is the one-chord diagram } \\
\operatorname{RootIns}_{i+\ell}\left(\operatorname{DiagIns}_{\widehat{C_{1}}, i}\left(C_{2}\right)\right) \text { if } C_{1} \text { is of the form } \operatorname{RootIns}\left(\widehat{C_{1}}\right)
\end{gathered}
$$

Decomposition according to this variant of the indexed product is known as the rootshare decomposition in $[22,15]$.

Note that this product gives the same recurrence of ordinary generating functions as the $\star$ product. The $\star$ product is combinatorially more convenient, particularly for the 


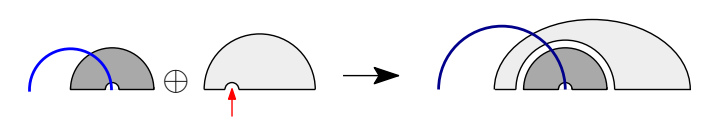

Figure 17: Schematic of the variant indexed product or root-share decomposition.

asymptotic counting of [10], while the $\oplus$ product is what was originally used in [22] and [15]. The two different products clearly give a permutation $\iota$ of the set of the connected diagrams: it takes the 1-chord diagram to itself, and otherwise for a connected diagram $C=C_{1} \star_{i} C_{2}$, it lets $\iota(C)=\iota\left(C_{1}\right) \oplus_{i} \iota\left(C_{2}\right)$.

The constructions below use the $\oplus$ product so as to align with the original definitions from [22] and [15], but an analogous theory could be worked out from the $\star$ product.

The origin of the next definition is to carve out a class of rooted planar binary trees satisfying the same recurrence as the one that comes from either connected diagram product.

Definition 35 (Tree $\boldsymbol{\tau}(\mathrm{C})$ ). The map $\tau$ from connected diagrams to rooted planar binary trees with labeled leaves is defined as follows. The leaves of the tree correspond to the chords of the diagram; this correspondence is indicated by labeling the leaves by the indices of the chords in intersection order.

- The image of the one-chord diagram under $\tau$ is the rooted binary tree with one node. This node is a leaf and is labeled 1.

- Suppose $C$ is a connected chord diagram with at least 2 chords. Write $C=C_{1} \oplus_{k} C_{2}$. Let $T_{1}=\tau\left(C_{1}\right)$ and $T_{2}=\tau\left(C_{2}\right)$. Let $v$ be the $k$ th vertex of $T_{2}$ in a pre-order traversal. Let $T$ be the binary rooted tree obtained by beginning with $T_{2}$ and replacing $v$ with a new vertex which has the subtree rooted at $v$ as its right child and $T_{1}$ as its left child. Relabel the leaves of $T$ to correspond to the same chords but as indexed in $C$, that is, the leaf 1 from $T_{1}$ remains 1 , next come all the leaves of $T_{2}$ maintaining their relative order, and finally come all the other leaves of $T_{1}$ maintaining their relative order.

See Figure 18 for two examples; see [22, 15] for many more examples.

It turns out that $\tau$ is one-to-one, though describing the inverse map is tricky, and the best characterization we have for the image of $\tau$ is rather complicated (see [22]). Nonetheless, $\tau$ does have some nice properties. By construction, leaves correspond to chords under $\tau$ and vertices (including leaves) correspond to intervals. Furthermore, these trees can see the $\nu$ parameter, and the most natural decomposition of trees - the decomposition into the root along with the left and right subtrees - gives the formula (16) below.

Now we are ready for the original definition of $\nu$ (see [15] for more information on $\nu$ ).

Definition 36 (Parameter $\boldsymbol{\nu}(\boldsymbol{c})$ ). Let $C$ be a connected diagram and let $c$ be a chord of $C$. Let $\nu(c)$ be the length of the path which begins at the leaf of $\tau(C)$ associated to $c$ and goes up and to the left as far as possible. If this leaf is a left child, then $\nu(c)=0$. 

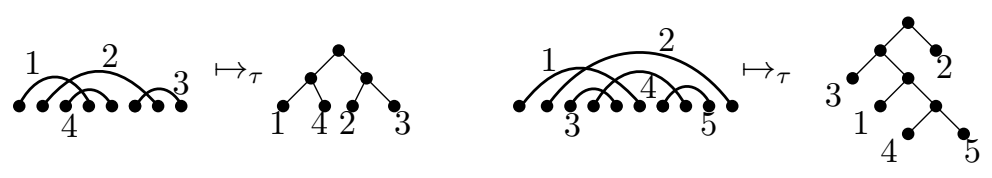

Figure 18: An example of the action of $\tau$.

For the first tree in Figure 18, $\nu(1)=\nu(2)=0, \nu(3)=2$ and $\nu(4)=1$ agreeing with what was used in Example 33. For the second tree in Figure 18, $\nu(1)=0, \nu(2)=1$, $\nu(3)=0, \nu(4)=0$, and $\nu(5)=3$. At this stage it is not apparent what this parameter measures about the chord diagram.

We are finally ready for the promised mysterious formula. To prove the main results of [22] and [15] we needed formulas which come from decomposing the binary tree associated to a diagram into its left and right subtrees. Reversing this decomposition involves grafting the trees and shuffling some of their labels (see [15, Section 5] for this grafting, and the shuffling operation worked out in detail). We have no interpretation for the decomposition directly at the level of chord diagrams. The formula in its more refined version is $[15$, Proposition 6.10]:

$$
\begin{aligned}
\sum_{\substack{\|C\|=i+1 \\
b(C)=j+1 \\
\nu(b(C))=n}} \hat{w}(C) A(C)=\sum_{k=1}^{i} \sum_{\ell=1}^{j}\left(\begin{array}{l}
j \\
\ell
\end{array}\right) & \left(\sum_{\substack{\left\|D_{1}\right\|=k \\
b\left(D_{1}\right) \geqslant \ell}} w\left(D_{1}\right) a_{d\left(b\left(D_{1}\right)\right), b\left(D_{1}\right)-\ell} A\left(D_{1}\right)\right) \\
& \times\left(\sum_{\substack{\left\|D_{2}\right\|=i-k+1 \\
b\left(D_{2}\right)=j-\ell+1 \\
\nu\left(b\left(D_{2}\right)\right)=n-1}} \hat{w}\left(D_{2}\right) A\left(D_{2}\right)\right)
\end{aligned}
$$

where

$$
\hat{w}(C)=\prod_{m \neq b(C)}\left(\begin{array}{c}
d(m) s+\nu(m)-2 \\
\nu(m)
\end{array}\right)=\frac{w(C)}{\left(\begin{array}{c}
d(b(C)) s+\nu(b(C))-2 \\
\nu(b(C))
\end{array}\right)} .
$$

We will give an interpretation of this equation in terms of maps in Subsection 5.7.

Notice that the first terminal chord $b(C)$ always has a special role to play in these quantum field theoretic chord diagram expansions. It has its own special factor in the solutions to the Dyson-Schwinger equations (9) and (13). In (16) on the left hand side we are ignoring the first terminal chord aside from fixing its size and index in the summation conditions. Then in the equation on the right hand side the first terminal chord of the subdiagrams in the second sum remains the first terminal chord in the whole diagram and so does not contribute outside the summation conditions, but the first terminal chord of the subdiagrams in the first sum becomes a later terminal chord in the whole diagram and so it contributes a factor and more possibilities of first terminal chord must be summed over. 


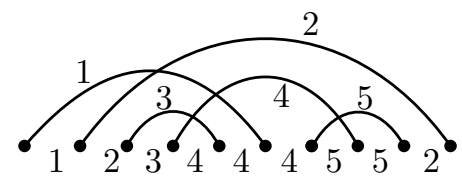

Figure 19: Covering numbers for the intersection order.

To go towards our new interpretation we need to associate numbers to chords in a more natural way, which the next subsection does.

\subsection{New interpretations on chord diagrams of the quantum field theoretic parameters}

In this subsection, we describe an alternative notion of $\nu$-index, which we call the covering number or $\omega$-index and which is more meaningful at the chord diagram level, while still satisfying the above formulas. This new notion is not equivalent to the old one, so we have to establish some bijections to show that the statistics are indeed equidistributed.

Definition 37 (Covering number $\boldsymbol{\omega}(\boldsymbol{i}))$. Let $C$ be a connected diagram. Fix an order $c_{1}<\cdots<c_{n}$ for the chords of $C$ (for example the intersection order). Proceeding through all the chords of $C$ in that order, mark all the intervals below the current chord with the index of that chord, replacing any previous marks. At the end of this procedure, the intervals are partitioned among the chords according to their markings. For $i \in\{1, \ldots, n\}$, let $\omega(i)$ be the number of intervals labeled by $i$ in this way, minus 1 .

An example of this construction for the intersection order is given in Figure 19. For this diagram, we have $\omega(1)=\omega(3)=0, \omega(2)=\omega(5)=1, \omega(4)=2$. Note that $\nu$ and $\omega$ are not equal.

Proposition 38. If we change every occurrence of $\nu$ to $\omega$, then, for the intersection order, Equation (16) still holds, and the function $G(x, L)$ defined by (13) still solves (10).

The proof of the proposition directly derives from the following lemma which says that the number of diagrams with the same $\nu$ and $\omega$ vectors are equal. Moreover, this remains true if we fix the indices of the terminal chords for the intersection order.

Lemma 39. Let $n$ be an integer. Given an n-vector $\boldsymbol{v}=\left(v_{1}, \ldots, v_{n}\right)$, and a subset $S$ of $\{1, \ldots, n\}$, we denote by $A_{\boldsymbol{v}, S}$ (resp. $B_{\boldsymbol{v}, S}$ ) the set of connected diagrams of size $n$ such that the positions of the terminal chords for the intersection order are given by $S$, and such that $\nu(i)=v_{i}$ (resp. $\omega(i)=v_{i}$ ) for every $i \in\{1, \ldots, n\}$. Then, for every vector $\boldsymbol{v}$ and subset $S$, the cardinal of $A_{\boldsymbol{v}, S}$ is the same as $B_{\boldsymbol{v}, S}$.

For example, there are three connected diagrams with 3 chords and with only the last chord as a terminal chord. These diagrams are illustrated in Figure 20 with their values of $\nu$ and $\omega$ written as vectors along with the constructions to determine the vectors. Note that for both $\nu$ and $\omega$ there is one diagram corresponding to the vector $(0,1,1)$ and two corresponding to $(0,0,2)$ but which diagrams are which is not the same. 

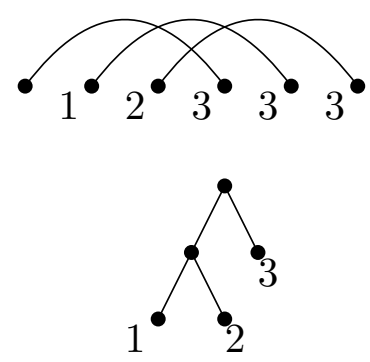

$$
\begin{aligned}
& \nu=(0,1,1) \\
& \omega=(0,0,2)
\end{aligned}
$$
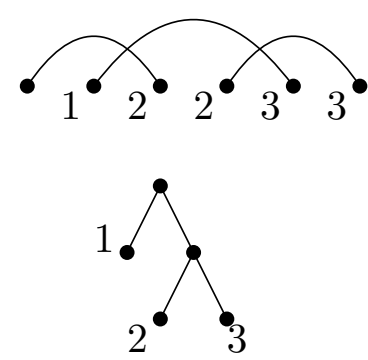

$\nu=(0,0,2)$

$\omega=(0,1,1)$
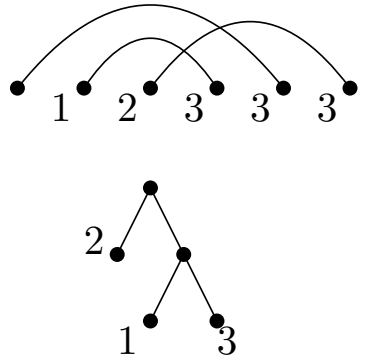

$\nu=(0,0,2)$

$\omega=(0,0,2)$

Figure 20: Connected diagrams on 3 chords with only the last chord terminal along with some associated information.

Proof. The proof is by induction on the number of chords. The result clearly holds for $n=1$.

Consider two vectors $\boldsymbol{u}=u_{1}, \ldots, u_{n_{1}}$ and $\boldsymbol{v}=v_{1}, \ldots, v_{n_{2}}$, and two subsets $S_{1} \subseteq$ $\left\{1, \ldots, n_{1}\right\}$ and $S_{2} \subseteq\left\{1, \ldots, n_{2}\right\}$. We suppose by induction that $\left|A_{\boldsymbol{u}, S_{1}}\right|=\left|B_{\boldsymbol{u}, S_{1}}\right|$ and $\left|A_{\boldsymbol{v}, S_{2}}\right|=\left|B_{\boldsymbol{v}, S_{2}}\right|$.

We are going to prove that the $\nu$-indices among the diagrams of the form $C_{1} \oplus_{k} C_{2}$ with $C_{1} \in A_{\boldsymbol{u}, S_{1}}, C_{2} \in A_{\boldsymbol{v}, S_{2}}$ and $k \in\{1, \ldots, n\}$, are distributed in the same way as the $\boldsymbol{\omega}$-indices among the diagrams of the form $C_{1}^{\prime} \oplus_{k} C_{2}^{\prime}$ with $C_{1}^{\prime} \in B_{\boldsymbol{u}, S_{1}}, C_{2}^{\prime} \in B_{\boldsymbol{v}, S_{2}}$ and $k \in\{1, \ldots, n\}$. The induction will then be shown by summing over all vectors $\boldsymbol{u}, \boldsymbol{v}$ and subsets $S_{1}, S_{2}$ such that $n_{1}+n_{2}=n$. Remark that in diagrams of the form $C=C_{1} \oplus_{k} C_{2}$, the positions of the terminal chords in $C$ for the intersection order only depend on $S_{1}$ and $S_{2}$; this is why we only need to focus on the $\nu$-indices and the $\omega$-indices.

Fix $C_{1} \in A_{\boldsymbol{u}, S_{1}}$ and $C_{2} \in A_{\boldsymbol{v}, S_{2}}$. When constructing $\tau\left(C_{1} \oplus_{k} C_{2}\right)$ from $\tau\left(C_{1}\right)$ and $\tau\left(C_{2}\right)$, we add a new vertex along one of the leftwards paths, so we increase exactly one $\nu$ index by 1 . Furthermore, running over all $k$ means performing this path lengthening once at each vertex of $\tau\left(C_{2}\right)$. We can more precisely observe that, for every $i \in\left\{1, \ldots, n_{2}\right\}$, there are $v_{i}+1$ possibilities among the choices of $k$ to increase $\nu(i)$ by 1 , since, by definition, the leftward path starting at the leaf labeled by $i$ contains $v_{i}+1$ vertices. Eventually, we notice that for every vector $\boldsymbol{w}$ of the form $\left(u_{1}=0, v_{1}, \ldots, v_{i-1}, v_{i}+\right.$ $\left.1, v_{i+1} \ldots, v_{n_{2}}, u_{2}, \ldots, u_{n_{1}}\right)$, the set $A_{\boldsymbol{w}, S}$ contains exactly $v_{i}+1$ diagrams of the form $C_{1} \oplus_{k} C_{2}$, and zero such diagrams if $\boldsymbol{w}$ has a different form.

Now consider $C=C_{1}^{\prime} \oplus_{k} C_{2}^{\prime}$ where $C_{1}^{\prime} \in B_{\boldsymbol{u}, S_{1}}$ and $C_{2}^{\prime} \in B_{\boldsymbol{v}, S_{2}}$ are fixed. For the intersection order of $C$, every non-root chord of $C_{1}^{\prime}$ comes after any chord of $C_{2}^{\prime}$. Thus, since the non-root chords of $C_{1}^{\prime}$ are below every chord of $C_{2}^{\prime}$, the marking of the intervals of $C_{1}^{\prime}$ (except the first one) will overwrite the marking of the intervals delimited by the chord of $C_{2}^{\prime}$. So, except a priori for the root chord, the $\omega$-index associated to the chords of $C_{1}^{\prime}$ will remain unchanged in $C$. However the $\omega$-index for the root chord is always 0 , because the label of every interval below the root chord other than the first one will be 
overwritten by other chords of $C$.

Concerning the intervals delimited by $C_{2}^{\prime}$, the marking will be unchanged except for the $k$ th leftmost interval of $C_{2}^{\prime}$, where the insertion of $C_{1}^{\prime}$ occurred, splitting this interval in two. The marking from the non-root chords of $C_{1}^{\prime}$ will occur and this will overwrite all the labels inserted into interval $k$, leaving just the two ends to be marked as the $k$ th interval was in $C_{2}^{\prime}$. So if the label of the $k$ th interval was $i$, then $\omega(i)$ will be increased by 1 and this is the only value of $\omega$ that changes. But, as we run over $k$, there are exactly $v_{i}+1$ intervals labeled by $i$ in $C_{2}^{\prime}$. Therefore, for every vector $\boldsymbol{w}$ of the form $\left(0, v_{1}, \ldots, v_{i-1}, v_{i}+\right.$ $\left.1, v_{i+1} \ldots, v_{n_{2}}, u_{2}, \ldots, u_{n_{1}}\right)$, the set $B_{\boldsymbol{w}, S}$ contains exactly $v_{i}+1$ diagrams of the form $C_{1}^{\prime} \oplus_{k} C_{2}^{\prime}$, and zero such diagrams if $\boldsymbol{w}$ has a different form.

Comparing the results for $C_{1} \oplus_{k} C_{2}$ and $C_{1}^{\prime} \oplus_{k} C_{2}^{\prime}$ over all $k$ enables us to conclude.

The ideas of this last proof are closely related to some unpublished ideas of one of us along with Markus Hihn [13]. Lemma 39 enables us to have a direct proof of Proposition 38 .

Proof of Proposition 38. Lemma 39 tells us that the generating functions of connected chord diagrams counted by terminal chords and $\nu$ vectors is the same with $\omega$ vectors instead. An additional integer weight on each chord carries through the constructions with no changes. Examples of such generating functions then, with some very particular choices of functions of these parameters, are $G(x, L)$ and the sums appearing in (16), hence these formulas cannot tell the difference between $\nu$ and $\omega$.

Lemma 39 also proves a conjecture of Hihn [14, Section 3.2.1]:

Corollary 40. The number of chord diagrams $C$ with a fixed set of terminal chords and with $\nu(|C|)=m$ is equal to the number of chord diagrams with the same set of terminal chords and where the vertex in the intersection graph corresponding to the last chord has $m$ neighbors.

Proof. In the algorithm to build $\omega$, the last chord marks all the intervals under it and the number of intervals under a chord is one more than the number of chords it crosses. Therefore Hihn's conjecture exactly states that the number of chord diagrams $C$ with a fixed set of terminal chords and $\nu(|C|)=m$ is equal to the number of chord diagrams with the same set of terminal chords and $\omega(|C|)=m$. This statement is a corollary of Lemma 39.

Some of Hihn's attempts to prove the conjecture led to the arguments of [13] which were generalized into Lemma 39.

Using $\omega$ in place of $\nu$ makes the parameters of (16) more natural, but what about the desired interpretation of the formula as coming from a decomposition: what chord diagram construction builds a connected diagram out of two connected diagrams in $\left(\begin{array}{l}j \\ \ell\end{array}\right)$ ways, with $j$ and $\ell$ as in the formula? For the $\nu$-index, the binomial coefficient counted shuffles of a subset of the labels of $\tau(C)$. For $\omega$ the rooted maps will save the day: there we have a direct interpretation involving shuffling the edges around the root vertex, see 
Figure 26. Rooted maps are the one place where everything becomes relatively natural. To get there we need one last change of order on the chords.

\subsection{Changing the ordering of the chords}

The intersection order does not induce a nice natural description when it is transposed to the set of combinatorial maps via the bijection $\theta$. In this subsection, we describe a new ordering on the chords of an indecomposable diagram for which Formulas (13) and (16) still work, and have a simple interpretation in the world of maps.

Definition 41 (Peeling order). The peeling order of an indecomposable diagram $D$ is defined as follows.

- The root chord of $D$ is the first chord in the peeling order.

- Remove the root chord of $D$. The result is not necessarily indecomposable. Let $D_{1}, D_{2}, \ldots, D_{k}$ be the indecomposable diagrams we obtain from left to right.

- For the peeling order of $D$, after the root chord come all the chord of $D_{k}$ ordered recursively in the peeling order, then all the chords of $D_{k-1}$ ordered recursively, and so on.

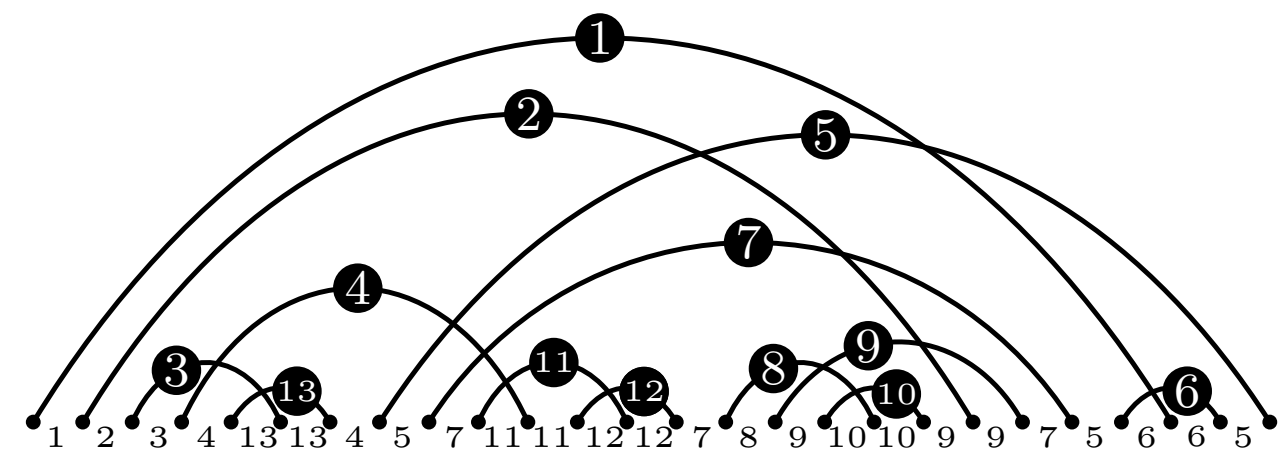

Figure 21: The peeling order of a connected diagram. The covering numbers are also indicated under the intervals.

An example of the peeling order is given by Figure 21. Although this example is connected, the peeling order on indecomposable but not connected diagrams comes up recursively. For example, upon removing the root chord of the diagram in Figure 21, we obtain a disconnected indecomposable diagram to which the definition of peeling order is applied.

Note that like the intersection order and the order by first endpoint, the peeling order extends the partial order on chords induced by the intersection graph.

Naturally, any connected diagram inherits a $\omega$-indexing from the peeling order. However, the vector distribution over all connected diagrams is not the same as for the in- 
tersection order ${ }^{5}$. Luckily, the parameters appearing in Equations (13) and (16) do not require the exact ordering of the chords, but weaker statistics, such as the multiset of the gaps between two consecutive terminal chords. It turns out that these weaker statistics agree for the intersection and the peeling order, implying that the quantum field theory formulas still hold for the peeling order. This also emphasizes that the gaps between terminal chords are the more natural chord diagram parameter rather than the indices of the terminal chords themselves.

Proposition 42. If we change every occurrence of $\nu$ to $\omega$, then, for the peeling order, Equation (16) still holds, and the function $G(x, L)$ defined by (13) still solves (10).

It will be helpful to consider an example before proceeding to the proof of the Proposition. Consider the statistics:

(1) the number of chords $|C|$, the sum of the chord weights $\|C\|$, the product

$$
\prod_{c \text { not terminal }} a_{d(c), 0}
$$

(which appears in the definition of $A(C)$ - see Equation (15));

(2) the position of the first terminal chord for the intersection order $b(C)$;

(3) the multiset formed by the pairs $(d(k), \omega(k))$, where $d(k)$ is the weight associated to the $k$ th chord in the intersection order, and $\omega(k)$ its covering number for the intersection order (used to define $w(C)$ - see Equation (14));

(4) the monomial $\alpha(C)=\prod_{j=2}^{\ell} a_{d\left(t_{j}\right), t_{j}-t_{j-1}}$, where $t_{1}=b(C)<t_{2}<\cdots<t_{\ell}=|C|$ lists the positions of all the terminal chords in intersection order.

The core of the proof of the proposition will be to show these statistics are preserved diagram by diagram when we replace the intersection order by the peeling order. Proposition 42 will be then a consequence of Proposition 38. We can first check it on an example.

Example 43. Consider the diagram of Figure 22 where we have put a weight 2 on chords with labels $5,6,8$ (for the intersection order) and a weight 1 on the remaining chords. We have

(1) $|C|=13,\|C\|=16, \prod_{c \text { not terminal }} a_{d(c), 0}=a_{1,0}^{7}$;

(2) $b(C)=5$;

(3) the multiset $\{(d(k), \omega(k))\}$ contains four times $(1,0)$, five times $(1,1)$, once $(1,2)$, once $(2,1)$, twice $(2,2)$;

\footnotetext{
${ }^{5}$ We have observed that a chord with a high $\omega$-index tends to be smaller in the intersection order than in the peeling order. For example, there are 2 more connected diagrams of size 6 such that $\omega(1)=\omega(2)=$ $\omega(3)=0, \omega(4)=\omega(5)=2, \omega(6)=1$ for the intersection order, compared with the peeling order.
} 
(4) $\alpha(C)=a_{1,1}^{3} a_{1,2} a_{2,1} a_{2,2}$.

We can then verify that the same diagram but with the peeling order (see Figure 21) satisfies the same equalities. However remark that the positions of the terminal chords differ between the peeling order and the intersection order (these positions are given by $5,6,7,9,10,12,13$ for the peeling order, and by $5,6,8,9,11,12,13$ for the intersection order).

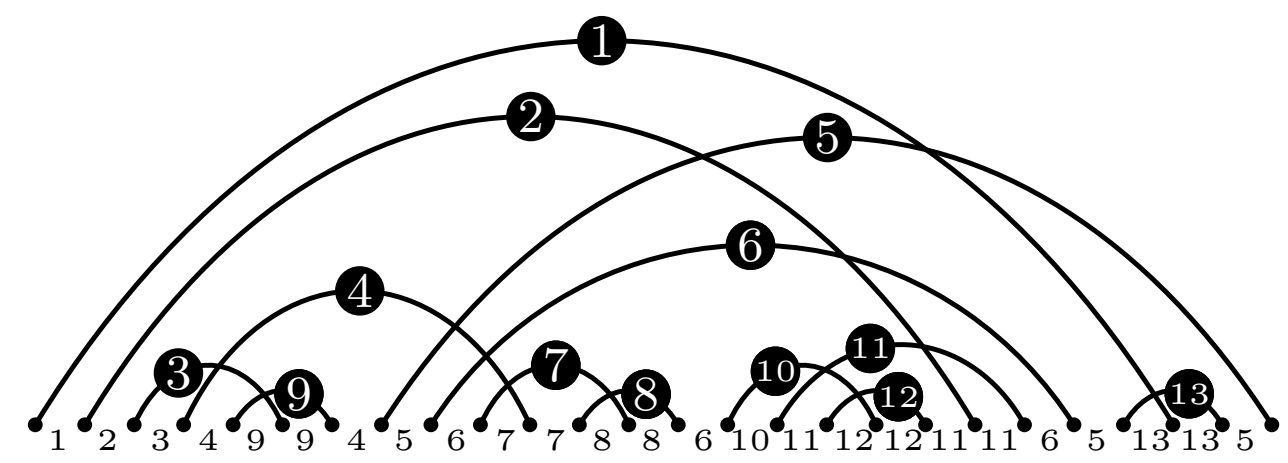

Figure 22: The intersection order version of the diagram of Figure 21.

We now proceed to the proof.

Proof of Proposition 42. Using Proposition 38, we saw that Formulas (16) and (13) only depend on the statistics on the connected diagrams $C$ that were enumerated above. We are going to prove that these statistics do not change if we swap the intersection order by the peeling order, which is sufficient to show the proposition.

Obviously, the statistics listed in (1) do not depend on the order.

As for the position of the first terminal chord given by (2), we can observe that the intersection order and the peeling order coincide for the first chords until the first terminal chord. Indeed, in both cases, after putting in first position the root chord and removing it, the first diagram we recursively sort is either the topmost connected component denoted by $C_{\uparrow}$ (for the intersection order), or the rightmost indecomposable diagram denoted by $D_{\rightarrow}$ (for the peeling order). The diagram $C_{\uparrow}$ is included in $D_{\rightarrow}$ and will be peeled first in $D_{\rightarrow}$ because the connected components below $C_{\uparrow}$ are to the left of the rightmost endpoint of $C_{\uparrow}$ (so they will appear at some point of the peeling of $D_{\rightarrow}$ to the left of what remains of $C_{\uparrow}$ ). Thus, the position of the first terminal chord remains the same for the intersection and peeling order.

Now let us consider the multiset $\{(d(k), \omega(k))\}_{k}$ described by (3). Remark that the covering number of a chord $c$ will only depend on the chords above/below $c$ in the diagram, and the chords intersecting $c$. But both for intersection and peeling order, a chord $c_{\downarrow}$ which is below a chord $c_{\uparrow}$ will satisfy $c_{\uparrow}<c_{\downarrow}$, while a chord $c_{\leftarrow}$ intersecting from the left a chord $c_{\rightarrow}$ will satisfy $c_{\leftarrow}<c_{\rightarrow}$. Therefore, the covering number associated to any chord will remain the same for the intersection and the peeling order, hence the equality of the multisets. 
The point (4) is the most delicate equality to establish. To remove the ambiguity, let $\alpha_{\text {inter }}(C)$ be the version of $\alpha(C)$ for the intersection order, and $\alpha_{\text {peel }}(C)$ be the one for the peeling order. We are going to prove by induction that $\alpha_{\text {inter }}(C)=\alpha_{\text {peel }}(C)$ for any connected diagram $C$. Since the base case is clear, we assume that $C$ has at least 2 chords. Let $C_{1}, C_{2}, i$ be such that $C=C_{1} \oplus_{i} C_{2}$. We assume that $C_{1}$ is not a single chord, since it is easy to conclude by induction in that case.

First we observe that, in the intersection order, each non-root chord of $C_{1}$ is after any chord of $C_{2}$ (by definition). So if $C_{2}$ exactly contains $j$ terminal chords, then the terminal chords with positions $t_{1}, t_{2}, \ldots, t_{j}$ in $C$ are in $C_{2}$ (diagram in which the terminal chords have positions $\left.t_{1}-1, t_{2}-1, \ldots, t_{j}-1\right)$, and the other ones are in $C_{1}$. Moreover, the last chord of a connected diagram for the intersection order is terminal, hence $t_{j}=\left|C_{2}\right|+1$. Additionally, if $t_{1}^{\prime}=b\left(C_{1}\right), \ldots, t_{k}^{\prime}$ denote the positions of the terminal chords in $C_{1}$, we can check that $t_{j+p}=t_{p}^{\prime}+\left|C_{2}\right|$ for $p \in\{1, \ldots, k\}$. Taking all this into account, we obtain

$$
\begin{aligned}
\alpha_{i n t e r}(C) & =\alpha_{i n t e r}\left(C_{2}\right) \times a_{d\left(t_{j+1}\right), t_{j+1}-t_{j}} \times \alpha_{i n t e r}\left(C_{1}\right) \\
& =a_{d\left(b\left(C_{1}\right)\right), b\left(C_{1}\right)-1} \alpha_{i n t e r}\left(C_{1}\right) \alpha_{i n t e r}\left(C_{2}\right) .
\end{aligned}
$$

Now let us consider the peeling order. Let $D$ be the diagram $C_{1}$ with its root chord removed. When we remove the root chord of $C$, the diagram $D$ is left somewhere in the diagram $C_{2}$. When we continue to peel $C$, the chords of $D$ will remain unconsidered until the point where $D$ appears as one of the indecomposable diagrams $D_{1}, D_{2}, \ldots, D_{k}$. There are then two possibilities: either $D=D_{k}$ and then the chord preceding the first chord of $D$ for the peeling order is a chord going over $D$ and ending at the rightmost point of the diagram; or $D=D_{j}$ with $j<k$ and then the chord preceding the first chord of $D$ is the last chord of $D_{j+1}$. In any case, the chord preceding the first chord of $D$ is terminal, so its position should be of the form $t_{q}$. Thus, if $t_{1}^{\prime}, \ldots, t_{k}^{\prime}$ denote the positions of the terminal chords of $D$, then $t_{q+r}=t_{r}^{\prime}+t_{q}$, for $r \in\{1, \ldots, k\}$. We have then

$$
\prod_{j=q+1}^{q+k} a_{d\left(t_{j+1}\right), t_{j+1}-t_{j}}=a_{d\left(t_{q+1}\right), t_{q+1}-t_{q}} \alpha_{p e e l}(D)=a_{d(b(D)), b(D)} \alpha_{p e e l}(D) .
$$

Furthermore, $C_{1}$ differs from $D$ just by a root chord insertion, hence we have $\alpha_{\text {peel }}(D)=$ $\alpha_{\text {peel }}\left(C_{1}\right)$ so that

$$
\prod_{j=q+1}^{q+k} a_{d\left(t_{j+1}\right), t_{j+1}-t_{j}}=a_{d\left(b\left(C_{1}\right)\right), b\left(C_{1}\right)-1} \alpha_{p e e l}\left(C_{1}\right) .
$$

Compare now the peelings of $C$ and $C_{2}$. We can process them in parallel, except that at some point in the peeling of $C$, we have to treat the subdiagram $D$. After finishing the peeling of $D$, we can resume the peeling of $C$ and $C_{2}$ in parallel. Thus, since the chord visited just before $D$ has label $t_{q}$, and $D$ has $k$ terminal chords, the set of gaps between two terminal chords of $C_{2}$ is constituted by $t_{2}-t_{1}, t_{3}-t_{2}, \ldots, t_{q}-t_{q-1}$ (occurring in $C$ before visiting $D$ ), then $t_{q+k+1}-|D|-t_{q}$ (in $C_{2}$ we do not visit $D$, so we have to subtract $|D|$ from the labels $\geqslant t_{q}+\left|C_{2}\right|$ of $C$ to recover the labels of $C_{2}$ ), and finally 
$t_{q+k+2}-t_{q+k+1}, \ldots, t_{\ell}-t_{\ell-1}$ (occurring in $C$ after $D$ ). Note that $t_{q+k}=t_{k}^{\prime}+t_{q}$, which is also equal to $|D|+t_{q}$ since the last chord is always terminal. Therefore we have

$$
\alpha_{\text {peel }}\left(C_{2}\right)=\prod_{j=1}^{q} a_{d\left(t_{j+1}\right), t_{j+1}-t_{j}} \times \prod_{j=q+k+1}^{\ell} a_{d\left(t_{j+1}\right), t_{j+1}-t_{j}}
$$

so that

$$
\alpha_{\text {peel }}(C)=a_{d\left(b\left(C_{1}\right)\right), b\left(C_{1}\right)-1} \alpha_{\text {peel }}\left(C_{1}\right) \alpha_{\text {peel }}\left(C_{2}\right) .
$$

We then conclude that $\alpha_{\text {inter }}(C)=\alpha_{\text {peel }}(C)$ by the induction hypothesis.

\subsection{Restating the quantum field theory formulas in terms of maps}

Now let us think about how all the previous work clarifies the situation when the diagrams are transformed into combinatorial maps under $\theta$.

The key is that here the orientation of the map given by the rightmost DFS (Depth First Search) of the map.

Definition 44 (Rightmost DFS). The principle of the rightmost DFS is the following. Starting from the root, we explore the map as far as possible by choosing at each newly visited vertex the nearest half-edge in clockwise order. If the other associated half-edge belongs to an already visited vertex, we backtrack. We stop once every edge has been visited.

This map traversal naturally gives an orientation of the edges of the map, as illustrated by Figure 23. Rightmost DFS also induces a spanning tree (the same as in the Bridge First labeling), which consists of edges that revealed new vertices.

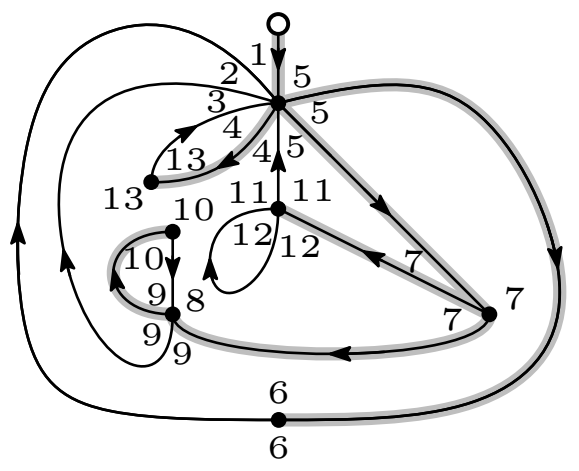

Figure 23: The rightmost DFS of a map and its associated statistics.

We now give an equivalent of the $\omega$-index for maps $M$. The principle is illustrated by Figure 24 .

Definition 45 (DFS-labeling of a map). We are going to label the corners of a map $M$ with integers $1, \ldots,|M|$, using the orientation induced by the rightmost DFS. We start 
with the corner following the root, whose label is 1 . Suppose that the current corner is labeled by $i$, and the next corner around the vertex in the counterclockwise order is not labeled. If the edge separating these two corners is ingoing, then we label the second corner by $i+1$; otherwise, the edge is outgoing, and we label the corner by $i$. Once all corners around the current vertex have been labeled, we go to the vertex which has been visited next during the rightmost DFS. Around this vertex, there is only one ingoing edge coming from the spanning tree induced by the rightmost DFS - it is the first edge that enabled the visit of this vertex. We then label the corner following this edge by the next available label, and continue the procedure. We stop when every corner is labeled.

The reader can refer again to Figure 23 for an example.

Similarly to diagrams, we can define $\omega(k)$ for maps as the number of corners carrying the label $k$ (minus 1). However it will be more convenient to define $\omega$ for edges. Thus, to each edge $e$, the integer $\omega(e)+1$ is the number of corners carrying the same label as the corner that is clockwisely adjacent to the ingoing part of $e$. Equivalently, $\omega(e)$ is the number of outgoing edges between the ingoing part of $e$ and the next ingoing half-edge after $e$ in the clockwise order. For example, the value of $\omega$ applied to the root of the map in Figure 23 is 2, since there are three labels 5.

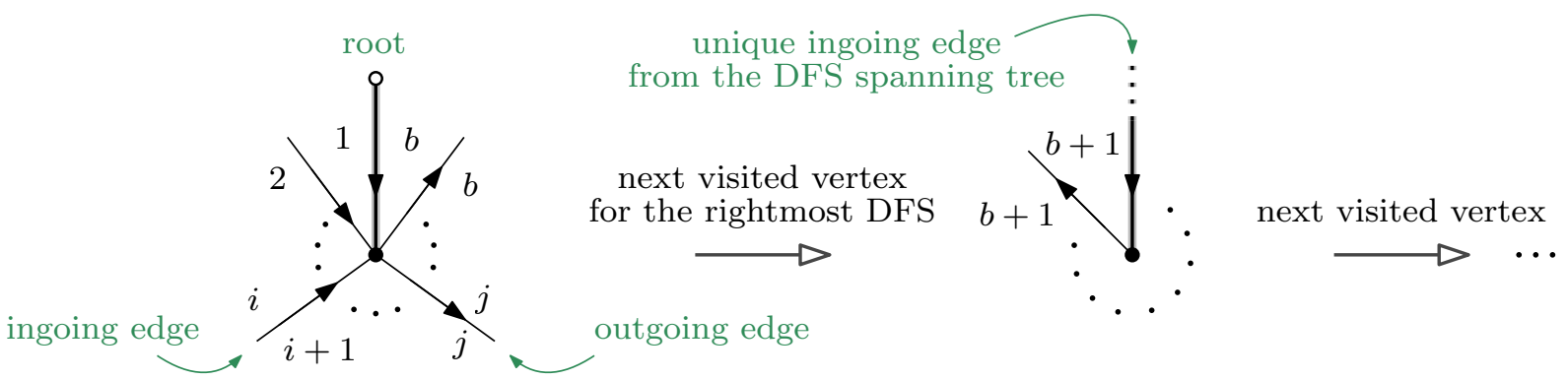

Figure 24: DFS-labeling procedure

We can now describe how the statistics from the QFT formulas translate to maps.

Proposition 46. Under the bijection of Section 4, the parameters of (13) are transferred as indicated by Table 3.

This proposition can be in particular verified by comparing Figures 21 and 23 (see Example 43 for the diagram parameters worked out), whose map and diagram are in bijection through $\phi$.

The most striking correspondence is the one between the terminal chords and the vertices of a map. First of all, it implies that the original QFT formulas can be expressed in terms of bridgeless maps counted with respect to edges and vertices, which are admittedly more natural than connected diagrams and terminal chords. It also again emphasizes that the gaps not the terminal chords themselves are the right parameter. Moreover, all the asymptotic results of [10] translate over to asymptotics about vertices of bridgeless maps. In particular, it implies the following. 


\begin{tabular}{c|c}
\hline Parameters in connected diagrams & Parameters in bridgeless maps \\
\hline \hline chords & edges \\
\hline terminal chords & $\begin{array}{c}\text { vertices; equivalently, edges in spanning } \\
\text { tree induced by the rightmost DFS }\end{array}$ \\
\hline gap $t_{j}-t_{j-1}$ between the $(j-1)$ th and \\
the $j$ th terminal chords & $\begin{array}{c}\text { number of ingoing edges (for the } \\
\text { rightmost DFS) incident to root vertex }\end{array}$ \\
\hline $\begin{array}{c}\text { number of ingoing edges (for the } \\
\text { rightmost DFS) incident to vertex } \\
\text { for the peeling order } k \text { th chord }\end{array}$ & $\begin{array}{c}\text { number of corners labeled by } k \\
\text { for DFS-labeling procedure minus } 1\end{array}$ \\
\hline
\end{tabular}

Table 3: How parameters in the QFT formulas transfer from diagrams to maps.

Corollary 47. The number of vertices in a random uniform bridgeless map with $n$ edges asymptotically obeys a Gaussian law of mean $\sim \ln n$ and of variance $\sim \ln n$.

Proof of Proposition 46 (sketch). The proof is a simple induction on (not necessarily bridgeless) maps $M$. It uses the fact that $\theta$ can be extended to $\phi$ (see Theorem 22). Indeed, it is sufficient to consider $M$ under all possible forms (map reduced to one edge; $\left.M=\operatorname{MapIns}_{M_{2}, 1}\left(M_{1}\right) ; M=\operatorname{RootIns}_{i}\left(M^{\prime}\right)\right)$ and confront it to its image under $\phi$ (respectively the diagram reduced to one chord; $\phi(M)=\operatorname{MapIns}_{\phi\left(M_{2}\right), 1}\left(\phi\left(M_{1}\right)\right)$; $\left.\phi(M)=\operatorname{RootIns}_{i}\left(\phi\left(M^{\prime}\right)\right)\right)$.

The proof is not difficult, but it requires a tedious checking through all parameters. All the necessary ideas are depicted in Figure 25.

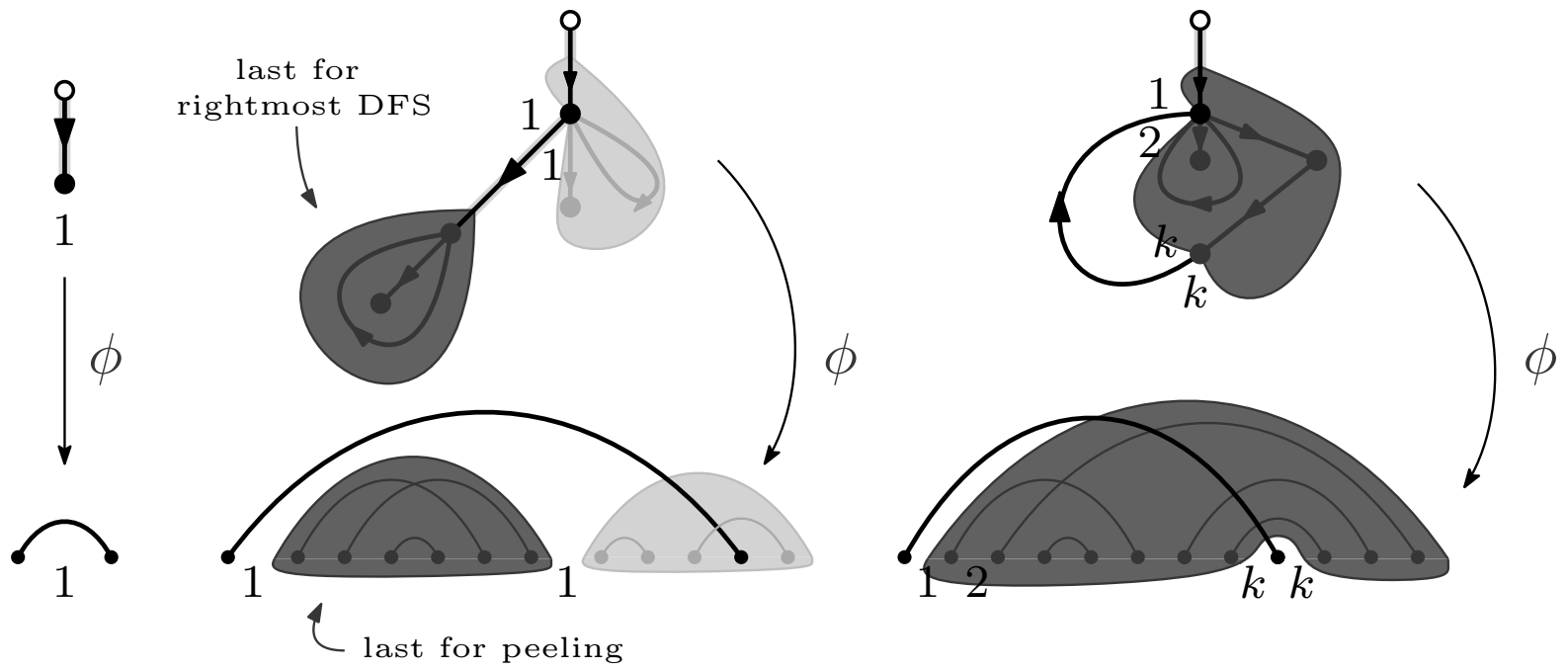

Figure 25: How the statistics evolve from maps to indecomposable diagrams 
Thanks to Propositions 42 and 46, we can rewrite the formulas we described in Subsection 5.2 in terms of maps, offering a new viewpoint on these equations. In particular, Equation (13) can be written under the following form.

Corollary 48. Let $F_{k}(\rho)$ be a formal Laurent series of the form $\sum_{i \geqslant 0} a_{k, i} \rho^{i-1}$, and $s$ be a positive integer parameter. The Dyson-Schwinger equation

$$
G(x, L)=1-\left[\rho^{0}\right] \sum_{k \geqslant 1} x^{k} G\left(x, \partial_{-\rho}\right)^{1-s k}\left(e^{-L \rho}-1\right) F_{k}(\rho)
$$

has for solution

$$
G(x, L)=1-\sum_{M}\left(\sum_{i=1}^{\operatorname{RootInDeg}(M)} a_{d(\operatorname{root}(M)), \operatorname{RootInDeg}(M)-i} \frac{(-L)^{i}}{i !}\right) w(M) A(M) x^{\|M\|},
$$

where the sum runs over all bridgeless maps $M$, carrying a positive integer weight $d(e)$ on

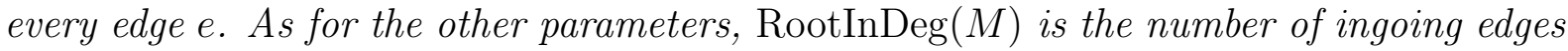
induced by the rightmost DFS (see Definition 44); $\|M\|$ is the sum of the edge weights;

$$
w(M)=\prod_{e \text { edge } \in M}\left(\begin{array}{c}
d(e) s+\omega(e)-2 \\
\omega(e)
\end{array}\right)
$$

$\omega(e)$ is the number of outgoing edges between the ingoing part of e and the next ingoing half-edge after $e$ in the clockwise order;

$$
A(M)=\prod_{\begin{array}{c}
\text { e not in the } \\
\text { nFS spanning tree }
\end{array}} a_{d(e), 0} \prod_{\begin{array}{c}
\text { efroot and in the } \\
\text { DFS spanning tree }
\end{array}} a_{d(e), \operatorname{InDeg}(\mathrm{v}(e))}
$$

and $\operatorname{InDeg}(\mathrm{v}(e))$ is the number of ingoing edges around the vertex pointed by the edge e.

Observe that in the case of the Dyson-Schwinger equation (8) the solution given above simplifies as we only sum over unweighted bridgeless maps, and $s=2$ so $w(M)=1$ in all cases. If we further take $F(\rho)=\rho /(1-\rho)$ then the coefficient of the $\left[L^{1}\right]$ part of the solution to the Dyson-Schwinger equation is the generating function of rooted connected chord diagrams, and the higher powers of $L$ give the generating functions of rooted connected chord diagrams where the position of the first terminal chord is at least the exponent of $L$. This was already observed empirically by Broadhurst and Kreimer in [7]. Translating this case through our bijection we obtain the generating function for bridgeless maps, (and for higher powers of $L$, generating functions for bridgeless maps with restrictions on the number of ingoing edges at the root), appearing in the solution to this Dyson-Schwinger equation. 


\subsection{A new combinatorial interpretation of a quantum field theoretic formula}

As an application of the map interpretation of the solution of the previous DysonSchwinger equations, we are going to describe an interpretation of Equation (16) at the map level. Then with Corollary 48 all steps and tools can be understood on the same objects namely combinatorial maps. Recall that this equation was in the core of the proof of the papers $[22,15]$ but the proof passed to rooted trees in an obscure way and was never understood at the level of chord diagrams. It can be reformulated in terms of maps as follows.

Theorem 49. Let $G_{d}(x, c)$ and $\widehat{G}_{d}(x, c)$ be the weighted generating functions

$$
\begin{aligned}
G_{d}(x, c) & \sum_{\begin{array}{c}
M \text { bridgeless map } \\
\text { with a weight }>0 \\
\text { on each edge } \\
\text { with RootInDeg }(M)=d
\end{array}} w(M) A(M) x^{\|M\|} c^{\omega(\operatorname{root}(M))}, \\
\widehat{G}_{d}(x, c) & \sum_{\begin{array}{c}
M \text { bridgeless map } \\
\text { with a weight }>0 \\
\text { on each edge } \\
\text { with RootInDeg }(M)=d
\end{array}} \hat{w}(M) A(M) x^{\|M\|} c^{\omega(\operatorname{root}(M))},
\end{aligned}
$$

where $\operatorname{RootInDeg}(M)$ is the number of ingoing edges induced by the rightmost DFS incident to the root vertex, $\|M\|$ is the sum of the edge weights, $\omega(\operatorname{root}(M))$ is the number of outgoing edges between the root and the next ingoing edge for the clockwise order, $w(M)$ and $A(M)$ are respectively defined by (17) and (18), and

$$
\hat{w}(M)=\prod_{\begin{array}{c}
e \text { edge } \in M \\
\text { different from the root }
\end{array}}\left(\begin{array}{c}
d(e) s+\omega(e)-2 \\
\omega(e)
\end{array}\right) .
$$

Then for $d \geqslant 2$,

$$
\widehat{G}_{d}(x, c)=c \sum_{\substack{d_{1} \geqslant 1, i \geqslant 1 \\
d_{1}+i=d}} \sum_{d_{2} \geqslant i}\left(\begin{array}{c}
d_{1}+i-1 \\
i
\end{array}\right) \widehat{G}_{d_{1}}(x, c) d e c_{d_{2}, i}(x) G_{d_{2}}(x, 1),
$$

where $\operatorname{dec}_{d_{2}, i}(x)=\sum_{k \geqslant 1} a_{k, d_{2}-i}(1-x) x^{k-1}$.

Proof. 1. Principle. This proof is rather complex and will be divided in several parts. The idea is to interpret the right side of Equation (19) as the combination of two bridgeless maps that we shuffle at the level of their root vertices.

More precisely, we are going to consider two bridgeless maps $M_{1}$ and $M_{2}$, where the numbers of ingoing edges (for the rightmost DFS) incident to the root vertex are respectively $d_{1}$ and $d_{2}$ and we fix any $i \in\left\{1, \ldots, d_{2}\right\}$. Roughly speaking, we are going to split the root vertex of $M_{2}$ in $d_{2}$ pieces containing each one of them an ingoing edge, then select the first $i$ such pieces and glue them on the root vertex on $M_{1}$. Meanwhile, 

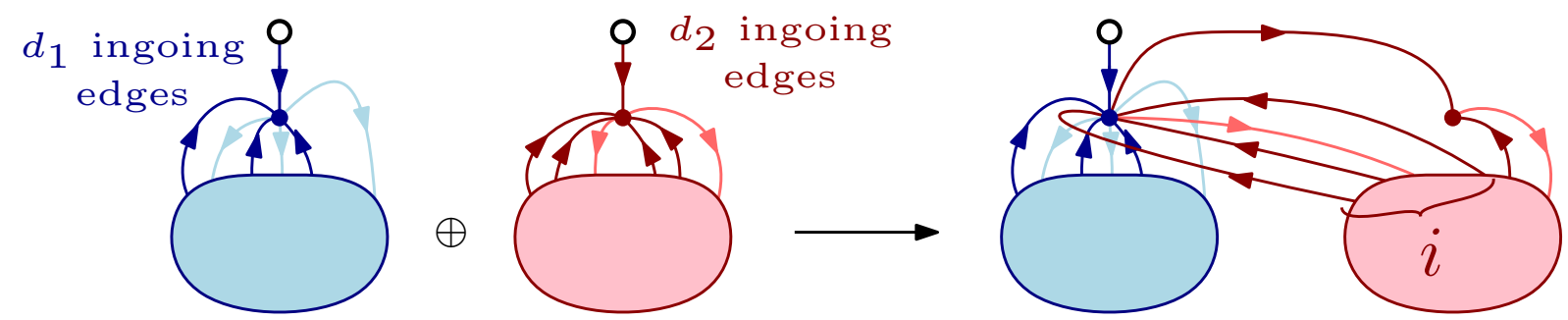

Figure 26: Interpretation of Equation (19) as the combination of two bridgeless maps.

the root of $M_{2}$ will be inserted at the corner just to the right of the root. This principle is illustrated by Figure 26.

The series $\operatorname{dec}_{d_{2}, i}(x)=\sum_{k \geqslant 1} a_{k, d_{2}-i}(1-x) x^{k-1}$ is introduced to deal with the fact that the root of $M_{2}$ is no longer the root after the operation, and so $A\left(M_{2}\right)$ has been modified.

2. Splitting the root of $\boldsymbol{M}_{\mathbf{2}}$. The half-edges incident to the root of $M_{2}$ can be listed in the counterclockwise order as

$$
i_{1},\left(o_{2,1}, \ldots, o_{2, j_{2}}\right), i_{2},\left(o_{3,1}, \ldots, o_{3, j_{3}}\right), i_{3}, \ldots,\left(o_{d_{2}, 1}, \ldots, o_{d_{2}, j_{d_{2}}}\right), i_{d_{2}}=\operatorname{root}\left(M_{2}\right),
$$

where $i_{1}, \ldots, i_{d_{2}}$ are the $d_{2}$ ingoing edges incident to the root vertex, $i_{d_{2}}$ is the root of $M_{2}$, and $\left(o_{k, 1}, \ldots, o_{k, j_{k}}\right)$ is the sequence (potentially empty) of outgoing edges preceding $i_{k}$. Note that $j_{k}=\omega\left(i_{k}\right)$ for every $k \in\left\{1, \ldots, d_{2}\right\}$.

We split the root vertex of $M_{2}$ into $d_{2}$ smaller vertices $v_{1}, \ldots, v_{k}$ such that the incident half-edges of $v_{k}$ are $o_{k, 1}, \ldots, o_{k, j_{k}}, i_{k}$. Let us denote the resulting map $\widehat{M}_{2}$. Remark that $\widehat{M}_{2}$ is still connected since we can still carry out a DFS with the same orientation (maybe not in the same order, but if we need to backtrack to the root vertex to follow an outgoing edge, this edge is necessarily attached to an ingoing edge which has been previously visited). The process is shown in Figure 27.
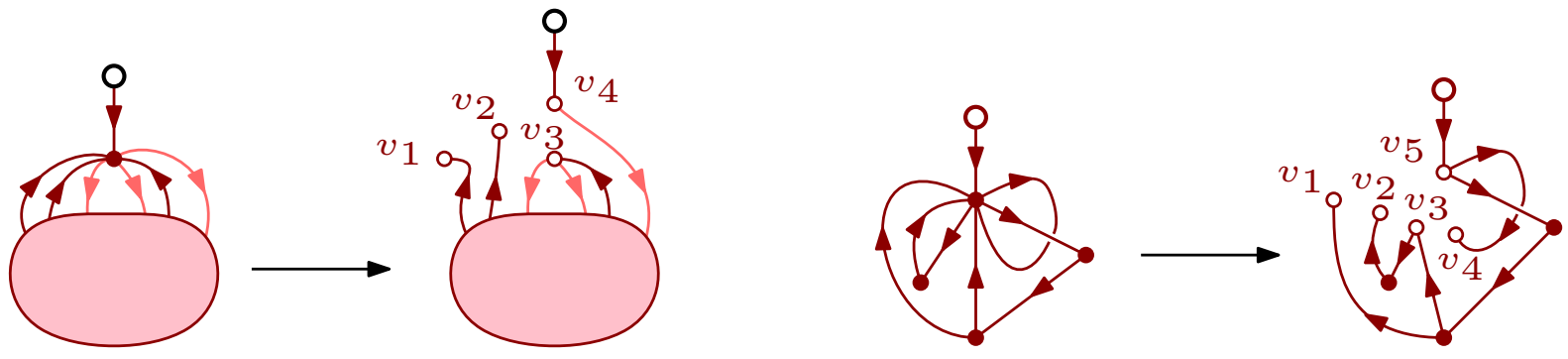

Figure 27: Typical splitting of $M_{2}$, along with an example.

3. Defining the map $\boldsymbol{M}$. We are going to merge the vertices $v_{1}, \ldots, v_{i}$ of $\widehat{M}_{2}$ with the root vertex of $M_{1}$ at some particular locations. These locations are just inside the corners that counterclockwisely follow an ingoing edge. (Thus there are $d_{1}$ such corners.) Figure 28 illustrates that.

We fix now a subset $S$ of these locations, multiplicity allowed, of size $i$. (Since we authorize multiple occurrences of the same location, there are $\left(\left(\begin{array}{c}d_{1} \\ i\end{array}\right)\right)=\left(\begin{array}{c}d_{1}+i-1 \\ i\end{array}\right)$ such 

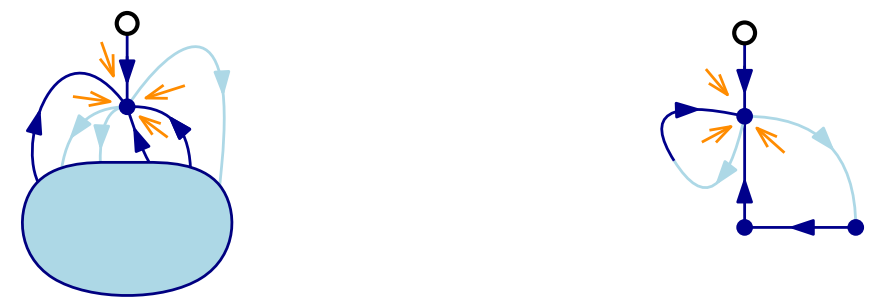

Figure 28: Corners after the ingoing edges, along with an example.

subsets.) Then we glue $v_{1}$ at the first ${ }^{6}$ corner given by $S$, putting $i_{1}$ in last. We similarly glue $v_{2}$ in the second position, then $v_{3}$, and so on and so forth, finishing by $v_{i}$. We glue back $v_{i+1}, \ldots, v_{d_{2}}$ as they were before in $M_{2}$.

Moreover, we attach the root of $\widehat{M}_{2}$ as a non-root edge just in the corner following the root of $M_{1}$ in the clockwise order.

The resulting map is denoted $M$. Complete examples are given by Table 4 . Note that when $i=d_{2}$, the root of $M_{2}$ becomes a loop.

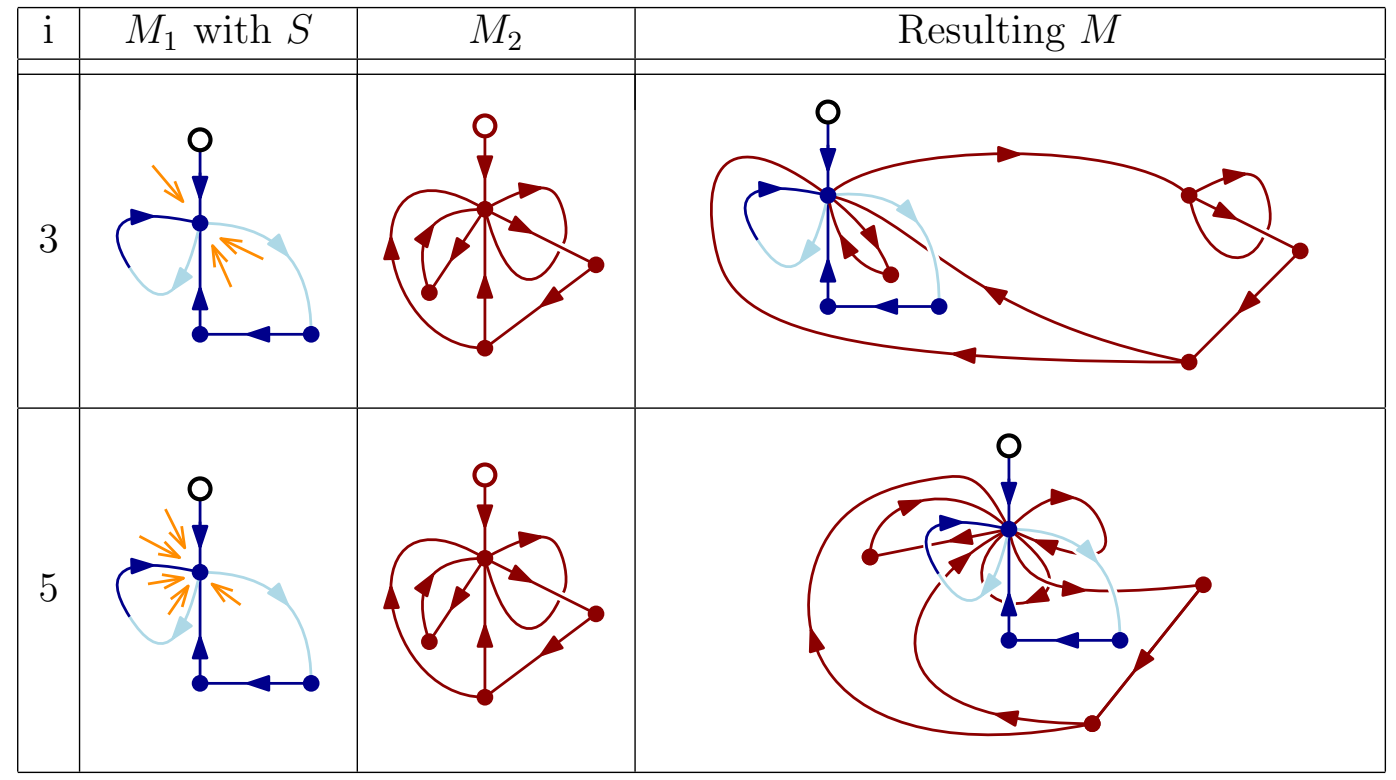

Table 4: Examples of combinations between two bridgeless maps $M_{1}$ and $M_{2}$.

4. How the parameters evolve. First of all, the weights on the edges do not change during the operation, so $\|M\|=\left\|M_{1}\right\|+\left\|M_{2}\right\|$.

One outgoing edge was added to the right of the root of $M$ (which was the root of $M_{2}$ ), so the number of outgoing edges of $M$ between the root and the next ingoing edge in the clockwise order has been increased by 1 compared to $M_{1}$. In other words, $\omega(\operatorname{root}(M))=\omega\left(\operatorname{root}\left(M_{1}\right)\right)+1$. Additionally, since each vertex $v_{1}, \ldots, v_{i}$ has one ingoing edge, we have $\operatorname{RootInDeg}(M)=\operatorname{RootInDeg}\left(M_{1}\right)+i=d_{1}+i$.

\footnotetext{
${ }^{6}$ First means here first in the counterclockwise order, if we start from the root.
} 
Concerning $A(M)$, we remark that it compiles every factor of $A\left(M_{1}\right)$ and $A\left(M_{2}\right)$, and the factor associated to the root of $M_{2}$ (which is no longer a root in $M$ ). There are two possibilities here: either $i<d_{2}$, and in that case, the root of $M_{2}$ belongs to the DFS spanning tree of $M$, and because we have removed $i$ ingoing edges to the root vertex of $M_{2}$, this factor is $a_{d\left(\operatorname{root}\left(M_{2}\right)\right), d_{2}-i}$; or $i=d_{2}$, and the root vertex of $M_{2}$ is merged with the root vertex of $M_{1}$, implying that $\operatorname{root}\left(M_{2}\right)$ is not in the spanning tree of $M$, hence the factor is $a_{d\left(\operatorname{root}\left(M_{2}\right)\right), 0}$. In every case, we have $A(M)=a_{d\left(\operatorname{root}\left(M_{2}\right)\right), d_{2}-i} A\left(M_{1}\right) A\left(M_{2}\right)$.

As for $\hat{w}(M)$, observe that $\omega(e)$ is invariant for every edge $e$ different from the root of $M$. We have for that purpose split the root of $M_{2}$ in pieces which preserve the number of outgoing edges before an ingoing edge. Consequently, $\hat{w}(M)=w\left(M_{1}\right) \hat{w}\left(M_{2}\right)$.

It is then relatively easy to see that the weighted generating function of maps $M$ (potentially with multiplicity) produced by the combinations of every pair of maps $M_{1}$ and $M_{2}$, with respectively $d_{1}$ and $d_{2}$ ingoing edges incident to the root vertex, is given by the right side of (19). The only subtlety here is the incorporation of $a_{d\left(\operatorname{root}\left(M_{2}\right)\right), d_{2}-i}$ which depends on the decoration of the root of $M_{2}$. To deal with this, we remark that the weighted generating function of maps $M_{2}$ where we have removed the weight of the root is given by $\frac{G_{d_{2}}}{\sum_{k \geqslant 1} x^{k}}=\frac{G_{d_{2}}}{\frac{x}{1-x}}$. Then, to recover the weight of the root of $M_{2}$ along with $a_{d\left(\operatorname{root}\left(M_{2}\right)\right), d_{2}-i}$, we have to multiply the previous series by $\sum_{k \geqslant 1} a_{k, d_{2}-i} x^{k}$, which gives $\operatorname{dec}_{d_{2}, i}(x) G_{d_{2}}$.

Thus, to prove Equation (19), it just remains to show that the construction is bijective, which is the purpose of the last point.

5. Recovering $\boldsymbol{M}_{1}, \boldsymbol{M}_{\mathbf{2}}$ and $\boldsymbol{i}$. Given a map $M$, we are going to construct two maps $M_{1}$ and $M_{2}$ whose combination gives $M$. The process is illustrated by Figure 29 .

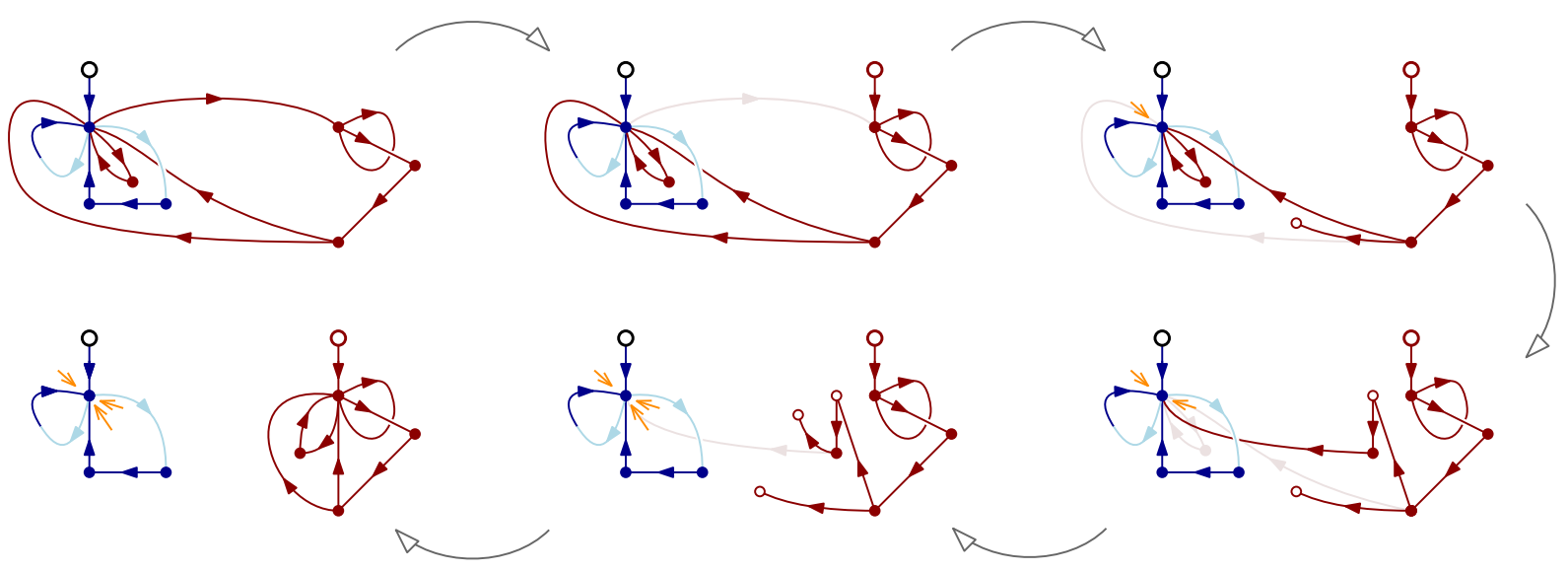

Figure 29: How to recover $M_{1}$ and $M_{2}$.

We start by detaching the edge clockwisely following the root edge and making it a root. This will be the root of the map $M_{2}$. We are going now to successively detach edges which are incident to the root vertex of $M$ until we obtain two separate maps.

To do so, we run a rightmost DFS of the map that starts from the root of $M_{2}$. Whenever we return to the root vertex of $M$, we detach the corresponding ingoing edge 
along with the whole sequence of outgoing edges that clockwisely follow it. We repeat this until $M_{2}$ forms a new connected component. At this point, we glue every detached vertex to the root vertex of $M_{2}$, in the same order that these vertices were attached to the root vertex of $M$.

We thus prove that the combination procedure is bijective.

Over all, the message here is that the map interpretation is helpful and more natural for the chord diagram expansions in quantum field theory of $[22,15,10]$. Some of these improvements are manifestly simple such as the reinterpretation of terminal chords as vertices. Others, such as the formula of this section, are considerably more intricate. Keep in mind, however, that the original proof of this formula was also very intricate and went though subtle auxiliary objects, and part of the complexity exhibited here is in proving the connection between the two approaches as in Subsection 5.5, rather than due to the new approach itself.

Aside from Equation (16), the other main ingredient in the original proof of (13) is another equation which expresses how the variant product decomposition of Definition 34 affects the sums over chord diagrams appearing in the solution $G(x, L)$. This becomes a recurrence for $\left[L^{k}\right] G(x, L)$ in terms of $\left[L^{i}\right] G(x, L)$ for $1 \leqslant i<k$, and corresponds to the renormalization group equation in physics. This should translate over straightforwardly to the bridgeless maps with the product decomposition that defines our bijection, and hence a proof of (13) entirely at the level of maps should be achievable, though the details have not been worked out.

\section{New interpretation of the Arquès-Béraud functional equation}

\subsection{Statement of the equation and implications}

In [2], Arquès and Béraud studied the two-variable generating function

$$
B(z, u)=u+z\left(u+u^{2}\right)+z^{2}\left(3 u+5 u^{2}+2 u^{3}\right)+z^{3}\left(15 u+32 u^{2}+22 u^{3}+5 u^{4}\right)+\cdots
$$

counting rooted maps with respect to $\operatorname{edges}^{7}(z)$ and vertices $(u)$, and proved that it satisfies the following simple functional equation:

$$
B(z, u)=u+z B(z, u) B(z, u+1)
$$

Arquès and Béraud showed how to derive (20) algebraically starting from another functional differential equation which they established through a root edge decomposition of maps on oriented surfaces of arbitrary genus (a refinement of the basic analysis we described in Section 2.2). Later, Cori [8] gave an alternative proof of (20) that made use of Ossona de Mendez and Rosenstiehl's bijection (henceforth, the "OMR bijection") between combinatorial maps and indecomposable involutions [25], which sends vertices of a map to

\footnotetext{
${ }^{7}$ Note that our rooting convention for maps allocates one additional (dangling) edge relative to Arquès and Béraud's convention, explaining the seeming shift by a factor of $z$.
} 
left-to-right maxima of the corresponding indecomposable involution. Speaking in terms of chord diagrams, left-to-right maxima correspond to top chords: that is, chords which are not below another chord. For example, the number of top chords in the diagrams of size 3 of Tables 1 and 2 are respectively 3, 3, 2, and 2 for the connected diagrams, and 1 , $1,1,2,2$, and 2 for the disconnected diagrams.

In the following section, we give a direct bijective interpretation of (20) on indecomposable chord diagrams. Besides its intrinsic interest, this bijection has the useful property that it restricts to connected diagrams to verify a modified functional equation:

$$
C(z, u)=u+z C(z, u)(C(z, u+1)-C(z, 1))
$$

By Theorem 10, we know that $C(z, 1)$ is also the generating function for bridgeless maps counted by number of edges, and we will use this fact later to derive an interesting application to the combinatorics of lambda calculus (Section 6.3). On the other hand, we do not see an obvious interpretation of the $u$ parameter of (21) for bridgeless maps: in particular, it is easy to check (by simple inspection of Table 1) that the coefficient of $z^{n} u^{k}$ in

$$
C(z, u)=u+z u^{2}+z^{2}\left(2 u^{2}+2 u^{3}\right)+z^{3}\left(10 u^{2}+12 u^{3}+5 u^{4}\right)+\cdots
$$

does not give the number of bridgeless maps with $n$ edges and $k$ vertices. This can also be seen as an explanation for why the OMR bijection cannot possibly restrict to a bijection between bridgeless maps and connected diagrams. Indeed, we have the following somewhat curious situation:

1. The OMR bijection sends vertices to top chords, but does not restrict to a bijection between bridgeless maps and connected diagrams.

2. The $\phi$ bijection of Section 4 restricts to a bijection between bridgeless maps and connected diagrams, but sends vertices to terminal chords rather than to top chords (see Proposition 46).

Taking either the $\phi$ bijection or the OMR bijection as a starting point leads naturally to two different open questions:

Question 50. Is there a natural statistic $Q$ of maps, such that the coefficient of $z^{n} u^{k}$ in (21) counts bridgeless maps with $n$ edges and $Q=k$ ?

Question 51. Is there a natural property $P$ of maps, such that the coefficient of $z^{n} u^{k}$ in (21) counts $P$-maps with $n$ edges and $k$ vertices?

Furthermore, we can state at this point another interesting phenomenon. Combining Observations 1 and 2 from above shows that the number of indecomposable diagrams with $n$ chords and $k$ terminal chords is equal to the number of indecomposable diagrams with $n$ chords and $k$ top chords, indeed that there is a bijection between these sets of diagrams given by composing $\phi$ with the inverse of the OMR bijection. In actual fact, the statistics counting terminal chords and top chords are more than equidistributed for indecomposable diagrams; they are symmetric: 
Proposition 52 ([21]). Indecomposable diagrams of size $n$ with $k_{1}$ terminal chords and $k_{2}$ top chords are in bijection with indecomposable diagrams of size $n$ with $k_{2}$ terminal chords and $k_{1}$ top chords.

The proof of this result, which was communicated to the authors by Mathias Lepoutre [21], uses the fact that one can recursively change the position of the leftmost closing endpoint.

\subsection{Combinatorial interpretation}

Before describing the interpretation of Equations (20) and (21) on chord diagrams, we take the opportunity of refining them to keep track of the number of crossings.

Theorem 53. Let $B(z, u, v)$ be the ordinary generating function of indecomposable diagrams counted with respect to the number of chords minus one (z), the number of top chords (u) and the number of crossings (v). Similarly, let $C(z, u, v)$ be the generating function for connected diagrams with the same interpretation of the parameters. The following equations hold:

$$
\begin{aligned}
& B(z, u, v)=u+z B(z, 1+u v, v) B(z, u, v) \\
& C(z, u, v)=u+z(C(z, 1+u v, v)-C(z, 1, v)) C(z, u, v) .
\end{aligned}
$$

Proof. From a combinatorial point of view, Equation (20) says that every indecomposable diagram with a least two chords can be seen as the product of two indecomposable diagrams, one of which has a marked subset of top chords.

We start by describing the combination part, building a diagram from two smaller ones. Figure 30 gives an example of such a combination.

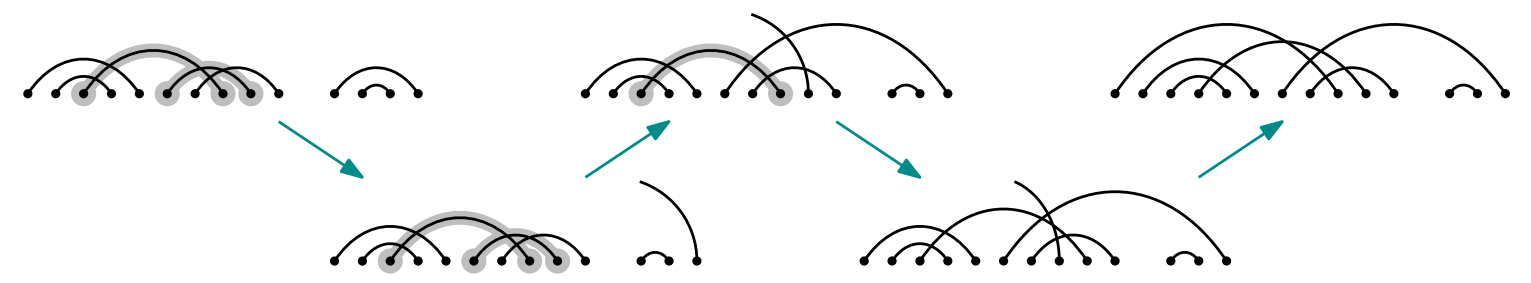

Figure 30: An example of how to combine an indecomposable diagram with another indecomposable diagram in which a subset of top chords is marked. The first diagram has 4 top chords, 2 of which are marked. The second diagram has only one top chord. The combination of both induces 3 top chords, as expected.

Let us thus consider two indecomposable diagrams $D_{1}$ and $D_{2}$, where some top chords of $D_{1}$ are marked. We run the following algorithm:

1. Put $D_{2}$ on the right of $D_{1}$. 
2. Open the left endpoint of the root chord $D_{2}$.

3. Consider the rightmost marked top chord. (The top chords are sorted from left to right without ambiguity.) If there are no more marked top chords, go to 5.

4. Forget the marking of that chord. Then, open its left endpoint, and replace it by the left endpoint of the other open arc. Go to 3.

5. Close the open arc at the left of $D_{1}$.

The composition of two diagrams is thus defined. We denote by $D$ the resulting diagram.

Let us enumerate the top chords of $D$. Each non-marked top chord of $D_{1}$ is now below a chord (which corresponds to the most immediate marked top chord to its right - or the root chord of $D_{2}$ if there were not any marked top chords on its right), so is not a top chord in $D$ anymore. On the contrary, each marked top chord of $D_{1}$ remains a top chord. Indeed, the only chords that change from $D_{1}$ to $D$ are the marked top chords, and the algorithm is constructed in such a way that a marked top chord never covers the marked top chords on its left. As for the other chords of $D$, it only takes a quick check to observe that non-top chords stay non-top chords, and top chords of $D_{2}$ stay top chords. Finally, the top chords of $D$ are given by the top chords of $D_{2}$ and the marked top chords of $D_{1}$.

As for the number of crossings in $D$, we can notice that the algorithm only creates crossings during the execution of step 4. Indeed, swapping an open arc and the left endpoint of a top chord (being on the left of the open arc) increases the number of crossings exactly by 1 . That is why the number of crossings in $D$ is the number of the crossings of $D_{1}$ and $D_{2}$, plus the number of marked top chords.

We just proved that the multi-set of diagrams $D$ induced by the combinations of diagrams $D_{1}$ and $D_{2}$ has for generating function $z B(z, 1+u v, v) B(z, u, v)$. To prove (22), we only need to show that our way of combining two diagrams to produce a larger diagram is bijective. For the inverse operation, we run the following algorithm, starting from an indecomposable diagram $D$ of size $>1$.

1. Open the left endpoint of the root chord of $D$.

2. If the resulting diagram is not indecomposable, go to 6 .

3. Consider the leftmost top chord intersecting the open arc.

4. Open its left endpoint, and replace it by the left endpoint of the other open arc.

5. Mark the chord that was just closed. Go to 2 .

6. Close the open arc to the right of the leftmost indecomposable component of $D$. We thus obtain two indecomposable diagrams $D_{1}$ (on the left) and $D_{2}$ (on the right).

To see that this algorithm computes an inverse to the first algorithm, the reader may refer again to Figure 30, which can be likewise read from right to left. This establishes that 
every indecomposable diagram which is not the one-chord diagram can be expressed as the combination of two diagrams, and so Equation (22) holds.

Note that a new connected component is created by this process if and only if no top chord is marked. Indeed, the only way to form a new component is to close the root chord of $D_{2}$ directly at the left of $D_{1}$, which can be done by jumping Item 4 . So if we want to enumerate connected diagrams, we have to force diagrams $D_{1}$ to have at least one marked top edge. Such diagrams are counted by $C(z, 1+u v, v)-C(z, 1, v)$. We recover Equation (23).

\subsection{An application to lambda calculus}

The results of the previous sections have a surprising application to the combinatorics of lambda calculus. As one of the authors described in [34], the original Arquès-Béraud equation (20) is also satisfied by the generating function counting certain natural isomorphism classes of terms in lambda calculus (namely, neutral linear terms modulo exchange of adjacent lambdas) by size and number of free variables. This fits a broader pattern of combinatorial connections recently discovered between different fragments of lambda calculus and different families of maps, beginning with a bijection between rooted trivalent maps and linear lambda terms found by Bodini, Gardy, and Jacquot [4], and a bijection between rooted planar maps and neutral planar lambda terms found by Giorgetti and Zeilberger [36]. It was also shown in [35] that the bijection of [4] restricts to a bijection between bridgeless (respectively, bridgeless planar) trivalent maps and linear (respectively, planar) lambda terms with no closed subterms - such terms were called "indecomposable" in [35], but here we call them unit-free to avoid confusion with indecomposable chord diagrams. Similarly, it is not difficult to check that the bijection of [36] restricts to a bijection between bridgeless planar maps and unit-free neutral planar terms. It is therefore tempting to draw the list of correspondences between families of lambda terms and families of rooted maps pictured in Table 5, where on the right we have indicated the index for the relevant OEIS entry counting objects by size (note that the size of a 3-valent map is defined here as its number of vertices, rather than edges).

The aforementioned works establish (either directly or as easy consequences) that each family of lambda terms is in the same combinatorial class as the corresponding family of rooted maps, for every row of Table 5 other than the boldfaced one. On the other hand, Proposition 12 above establishes that bridgeless maps are indeed counted by OEIS sequence A000699. So, to verify the full table, all that remains is to show that unit-free neutral linear terms (modulo exchange of adjacent lambdas) are counted by the same sequence.

Proposition 54 (cf. [34,35]). Let $C(z, u)$ be the two-variable generating function counting isomorphism classes of unit-free neutral linear lambda terms by size and number of free variables. Then $C(z, u)$ satisfies equation (21).

Proof. This is essentially immediate from definitions: see the references [34] and [35] for formal definitions of the relevant terms, as well as for the proofs of very similar equations. 


\begin{tabular}{llc} 
family of lambda terms & family of rooted maps & OEIS entry \\
\hline linear terms & 3-valent combinatorial maps & A062980 \\
planar terms & planar 3-valent maps & A002005 \\
unit-free linear terms & bridgeless 3-valent maps & A267827 \\
unit-free planar terms & bridgeless planar 3-valent maps & A000309 \\
neutral linear terms $/ \sim$ & combinatorial maps & A000698 \\
neutral planar terms & planar maps & A000168 \\
unit-free neutral linear $/ \sim$ & bridgeless maps & A000699 \\
unit-free neutral planar & bridgeless planar maps & A000260
\end{tabular}

Table 5: Known correspondences between different families of lambda terms and rooted maps. The correspondence in the boldfaced row was previously only conjectured, but is a corollary of our results here.

Corollary 55. Isomorphism classes of unit-free neutral linear lambda terms of size $n$ and with $k$ free variables are equinumerous with connected chord diagrams of size $n$ and with $k$ top chords.

Proof. Since by Proposition 54 and Theorem 53, their generating functions both satisfy the same equation (21).

Corollary 56. The number of isomorphism classes of unit-free neutral linear lambda terms of size $n$ is equal to the number of rooted bridgeless combinatorial maps of size $n$.

Proof. By combining Corollary 55 with Theorem 10 (or Proposition 12).

It is worth remarking that our proof of this enumerative result also implicitly yields a bijection between isomorphism classes of unit-free neutral linear lambda terms and rooted bridgeless combinatorial maps, by composing the bijection $\theta$ between bridgeless maps and connected diagrams with the implicit bijection between connected diagrams and this family of lambda terms that results from their admitting the same recursive decomposition (21). However, the meaning of this bijection is far less clear because we run into the obstacle posed by Question 50, namely, that it is not obvious what part of a rooted map should correspond to the free variables in a unit-free neutral linear term (i.e., what's counted by the $u$ parameter in $C(z, u))$. On the other hand, one might try to side-step this obstacle by passing directly from (bridgeless) combinatorial maps to (unitfree) neutral linear terms via an analogue of the bijection of Section 4. Given what we know about the transfer of statistics across that bijection (see Table 3), the following is therefore a natural related question.

Question 57. What (if anything) is the lambda calculus analogue for the terminal chords of a chord diagram? In particular, is there a natural invariant $Q$ of neutral linear terms, such that there is a bijection between connected diagrams of size $n$ with $k_{1}$ top chords and $k_{2}$ terminal chords, and isomorphism classes of unit-free neutral linear terms of size $n$ 
with $k_{1}$ free variables and $Q=k_{2}$ (cf. Corollary 55)? (A good notion of $Q$ should also be symmetrically distributed with the number of free variables among neutral linear terms of size $n$, following Proposition 52.)

\section{Conclusion}

After noticing an enumerative link between connected chord diagrams and bridgeless combinatorial maps, we made this observation into a size-preserving bijection $\theta$ by proving that these two families admit parallel decompositions in terms of an indexed product. An alternative decomposition based on root chord/root edge deletion then yielded another bijection $\phi$ between the larger families of indecomposable chord diagrams and rooted combinatorial maps, but these two bijections turned out to be essentially equivalent: $\theta$ is the restriction of $\phi$, while $\phi$ is the extension of $\theta$ obtained by composing with a "connected/bridgeless root component" decomposition. Moreover, we established that the bijection $\phi=\bar{\theta}$ has many other interesting properties as well: vertices correspond to terminal chords; planarity is equivalent to a forbidden pattern in the world of diagrams.

Some decompositions are apparently only meaningful for one of the two families, such as the decomposition of maps with respect to the number of ingoing edges for the rightmost DFS (Theorem 49), or the decomposition of diagrams with respect to the top chords (Theorem 53). On the other hand, since each of these decompositions describes interesting features for one of the combinatorial families, it is natural to wonder if they have analogues in the other class, highlighting new parameters (cf. Questions 50 and 51). There are other nice consequences of the present work which concern transversal areas, such as quantum field theory or lambda calculus. Indeed, our bijection between maps and diagrams has given interesting new perspectives on these domains and enabled a better understanding of some aspects of the theory, while suggesting a few natural directions for future work.

Finally, one may wonder about a non-recursive approach to a bijection between bridgeless maps and connected diagrams. Although the authors have thought in this direction and see no straightforward answer, it is not impossible that maps and diagrams conceal other nice connections.

\section{References}

[1] Huseyin Acan. An enumerative-probabilistic study of chord diagrams. PhD thesis, The Ohio State University, 2013.

[2] Didier Arquès and Jean-François Béraud. Rooted maps on orientable surfaces, Riccati's equation and continued fractions. Discrete Mathematics, 215:1-12, 2000.

[3] Olivier Bernardi. A characterization of the Tutte polynomial via combinatorial embedding. Annals of Combinatorics, 12(2):139-153, 2008.

[4] Olivier Bodini, Danielle Gardy, and Alice Jacquot. Asymptotics and random sampling for BCI and BCK lambda terms. Theoretical Computer Science, 502:227-238, 2013. 
[5] Béla Bollobás and Oliver Riordan. Linearized chord diagrams and an upper bound for Vassiliev invariants. Journal of Knot Theory and Its Ramifications, 09(07):847-853, 2000.

[6] David Broadhurst and Dirk Kreimer. Exact solutions of Dyson-Schwinger equations for iterated one-loop integrals and propagator-coupling duality. Nuclear Physics B, 600:403-422, 2001. arXiv:hep-th/0012146.

[7] D.J. Broadhurst and D. Kreimer. Combinatoric explosion of renormalization tamed by Hopf algebra: 30-loop Pade-Borel resummation. Physics Letters B, 475:63-70, 2000. arXiv:hep-th/9912093.

[8] Robert Cori. Indecomposable permutations, hypermaps and labeled Dyck paths. Journal of Combinatorial Theory, Series A, 116(8):1326-1343, 2009.

[9] Julien Courtiel and Karen Yeats. Next-to ${ }^{k}$ leading log expansions by chord diagrams. arXiv: 1906.05139.

[10] Julien Courtiel and Karen Yeats. Terminal chords in connected chord diagrams. Annales de l'Institut Henri Poincaré D, 4(4):417-452, 2017. arXiv:1603.08596.

[11] Bertrand Eynard. Counting Surfaces. Number 70 in Progress in Mathematical Physics. Birkhäuser, 2016.

[12] Philippe Flajolet, Jean Françon, and Jean Vuillemin. Sequence of operations analysis for dynamic data structures. Journal of Algorithms, 1(2):111 - 141, 1980.

[13] Markus Hihn. Personal communication.

[14] Markus Hihn. The Generalized Chord Diagram Expansion. PhD thesis, Humboldt Universität zu Berlin, 2014.

[15] Markus Hihn and Karen Yeats. Generalized chord diagram expansions of DysonSchwinger equations. arXiv:1602.02550.

[16] Ivo Hofacker, Peter Schuster, and Peter F. Stadler. Combinatorics of RNA secondary structures. Discrete Applied Mathematics, 88(1-3):207 - 237, 1998. Computational Molecular Biology DAM - CMB Series.

[17] Claude Itzykson and Jean-Bernard Zuber. Quantum Field Theory. McGraw-Hill, 1980. Dover edition 2005.

[18] David Jackson, Achim Kempf, and Alejandro Morales. A robust generalization of the Legendre transform for QFT. Journal of Physics A: Mathematical and Theoretical, 50(22), 2017. arXiv:1612.00462.

[19] Gareth A. Jones and David Singerman. Theory of maps on orientable surfaces. Proceedings of the London Mathematical Society, 37:273-307, 1978.

[20] Sergei K. Lando and Alexander K. Zvonkin. Graphs on Surfaces and Their Applications. Number 141 in Encyclopaedia of Math. Sciences. Springer-Verlag, 2004.

[21] Mathias Lepoutre. Personal communication. 
[22] Nicolas Marie and Karen Yeats. A chord diagram expansion coming from some Dyson-Schwinger equations. Communications in Number Theory and Physics, 7(2):251-291, 2013. arXiv:1210.5457.

[23] Albert Nijenhuis and Herbert. S. Wilf. The enumeration of connected graphs and linked diagrams. J. Combin. Theory A, 27:356-359, 1979.

[24] Patrice Ossona de Mendez and Pierre Rosenstiehl. Transitivity and connectivity of permutations. Combinatorica, 24(3):487-501, 2004.

[25] Patrice Ossona de Mendez and Pierre Rosenstiehl. Encoding pointed maps by double occurrence words. In University of Piraeus, editor, Volume of essays in honour of Professor Antonios C. Panayotopoulos, pages 701-712. Eptalofos, 2006.

[26] P. R. Stein. On a class of linked diagrams, I. Enumeration. Journal of Combinatorial Theory, Series A, 24:357-366, 1978.

[27] Alexander Stoimenow. On the number of chord diagrams. Discrete Mathematics, 218(1-3):209 - 233, 2000.

[28] Eric S. Swanson. A primer on functional methods and the Schwinger-Dyson equations. AIP Conference Proceedings, 1296:75-121, 2010. arXiv:1008.4337.

[29] Jacques Touchard. Sur un problème de configurations et sur les fractions continues. Canadian Journal of Mathematics, 4:2-25, 1952.

[30] Karen Yeats. Growth estimates for Dyson-Schwinger equations. PhD thesis, Boston University, 2008.

[31] Karen Yeats. Rearranging Dyson-Schwinger equations. Memoirs of the American Mathematical Society, 211, 2011.

[32] Karen Yeats. A combinatorial perspective on quantum field theory. Springer, 2017.

[33] Don Zagier. Vassiliev invariants and a strange identity related to the Dedekind etafunction. Topology, 40(5):945 - 960, 2001.

[34] Noam Zeilberger. Counting isomorphism classes of $\beta$-normal linear lambda terms. arXiv: 1509.07596.

[35] Noam Zeilberger. Linear lambda terms as invariants of rooted trivalent maps. Journal of Functional Programming, 26(e21), 2016.

[36] Noam Zeilberger and Alain Giorgetti. A correspondence between rooted planar maps and normal planar lambda terms. Logical Methods in Comp. Science, 11(3:22), 2015. 